\title{
Algebraic Markov equivalence for links in three-manifolds
}

\author{
S. Lambropoulou and C. P. Rourke
}

\begin{abstract}
Let $B_{n}$ denote the classical braid group on $n$ strands and let the mixed braid group $B_{m, n}$ be the subgroup of $B_{m+n}$ comprising braids for which the first $m$ strands form the identity braid. Let $B_{m, \infty}=\bigcup_{n} B_{m, n}$. We describe explicit algebraic moves on $B_{m, \infty}$ such that equivalence classes under these moves classify oriented links up to isotopy in a link complement or in a closed, connected, oriented three-manifold. The moves depend on a fixed link representing the manifold in $S^{3}$. More precisely, for link complements the moves are the two familiar moves of the classical Markov equivalence together with 'twisted' conjugation by certain loops $a_{i}$. This means premultiplication by $a_{i}^{-1}$ and postmultiplication by a 'combed' version of $a_{i}$. For closed three-manifolds there is an additional set of 'combed' band moves that correspond to sliding moves over the surgery link. The main tool in the proofs is the one-move Markov theorem using L-moves (adding in-box crossings). The resulting algebraic classification is a direct extension of the classical Markov theorem that classifies links in $S^{3}$ up to isotopy, and potentially leads to powerful new link invariants, which have been explored in special cases by the first author. It also provides a controlled range of isotopy moves, useful for studying skein modules of three-manifolds.
\end{abstract}

\section{Introduction and overview}

By a classic result of Brunn [Bru97] and Alexander [Ale23], any oriented knot in $S^{3}$ is isotopic to the closure of a braid and, by a theorem of Markov (and an improvement due to Weinberg) [Mar35, Wei39, Bir74], there is a bijection (induced by 'closing' the braid) between isotopy classes of oriented links and equivalence classes of braids, the equivalence being generated by braid isotopy and by two moves between braids: Markov conjugation (conjugating by a crossing) and the Markov move or $M$-move (adding an extra crossing at a rightmost point). In [LR97] a new type of braid move was introduced, the $L$-move (adding an in-box crossing; see Figure 5 below for abstract illustrations), and it was shown that the equivalence relation generated by $L$-moves and braid isotopy gives the same bijection. Consequently, Markov moves and Markov conjugation can be produced by $L$-moves (see Figures 13 and 14 below).

The Markov theorem can be regarded as a geometrical result (by thinking of braids as geometrical objects) or as an algebraic result (by thinking of braids as elements of the classical braid group $B_{n}$ ). In the latter case, the two moves of the Markov equivalence have the two well-known algebraic formulations. Similarly, the $L$-moves have analogous algebraic formulations (cf. [LR97, Remark 2.2]).

Received 23 September 2004, accepted in final form 10 January 2006.

2000 Mathematics Subject Classification 57M25, 57M27, 57N10.

Keywords: mixed links, mixed braids, knot complements, three-manifolds, braid equivalence, Markov equivalence, twisted conjugation, combed band move.

Most of this work was done while the first author held a position at the Laboratoire de Mathématiques Nicolas Oresme, Université de Caen.

This journal is (C) Foundation Compositio Mathematica 2006. 


\section{S. Lambropoulou and C. P. Rourke}
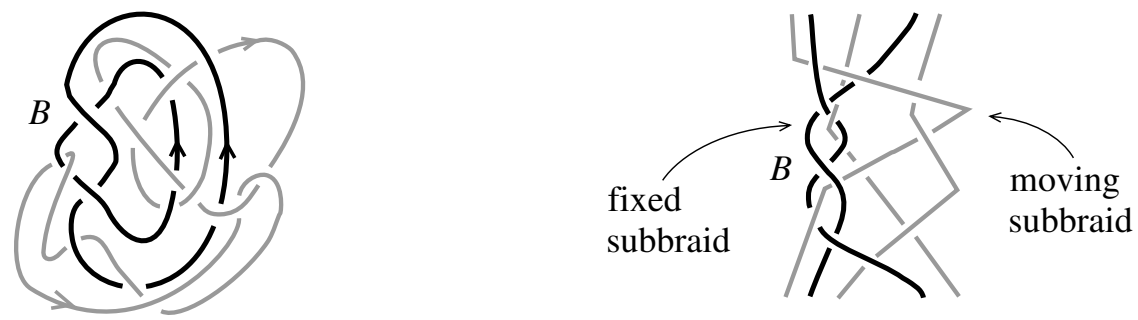

FiguRE 1. A mixed link and a mixed braid.

Now let $V$ be the complement of a link. By 'link complements' we mean complements of both knots and links and by 'links' we always mean knots and links. All links are considered oriented and piecewise linear (PL), but will be mostly illustrated as smooth for convenience. By the Alexander theorem, this link is isotopic to the closure $\widehat{B}$ of a braid $B$. So, we can write $V=S^{3} \backslash \widehat{B}$ and $V$ can be represented in $S^{3}$ by $\widehat{B}$. Further, let $V$ be a closed, connected, oriented three-manifold (we shall simply write 'closed three-manifold'). By classic results of Lickorish [Lic62] and Wallace [Wal60], $V$ can be obtained from $S^{3}$ by surgery along a framed link with integral framings. Without loss of generality, the surgery link can be assumed to be the closure $\widehat{B}$ of a surgery braid $B$. (Note that the framing of $\widehat{B}$ induces a framing on the surgery braid $B$.) So, we can write $V=\chi\left(S^{3}, \widehat{B}\right)$ and $V$ can be represented in $S^{3}$ by $\widehat{B}$. Moreover, by the proof in [Lic62], all components of the surgery link can be assumed unknotted and, as can be easily seen, they can be isotoped to the closure of a pure braid. Thus, for closed three-manifolds we may assume $B$ to be a pure braid.

Now let $L$ be an oriented link in $V=S^{3} \backslash \widehat{B}$ or $\chi\left(S^{3}, \widehat{B}\right)$. Fixing $\widehat{B}$ pointwise we may represent $L$ in $S^{3}$ unambiguously by the mixed link $\widehat{B} \cup L$, which consists of the fixed part $\widehat{B}$ and the 'moving' part $L$ that links with $\widehat{B}$ (see Figure 1 for an example). A mixed link diagram is a diagram $\widehat{B} \cup \widetilde{L}$ of $\widehat{B} \cup L$ on the plane of $\widehat{B}$. This plane is equipped with the top-to-bottom direction of $B$. By the Alexander theorem and as explained in [LR97, Theorem 5.3], a diagram $\widehat{B} \cup \widetilde{L}$ of $\widehat{B} \cup L$ may be turned into a mixed braid $B \cup \beta$ with isotopic closure. (The closure of a braid is obtained by joining each pair of corresponding endpoints by a simple arc.) This is a braid in $S^{3}$ with two different sets of strands, abstractly represented by a braid box with two differently coloured sets of strands. The point here is that one of the two sets comprises the fixed subbraid $B$ and not any other Markov equivalent one. The other set of strands representing the link in the manifold $V$ is called the moving subbraid. See Figure 1 for an example. So, $V$ may be represented in $S^{3}$ by the open braid $B$.

Consider now an isotopy of $L$ in $V$. It follows from standard results of PL topology that $L_{1}$ and $L_{2}$ are two instances of an isotopy in $S^{3} \backslash \widehat{B}$ if and only if the corresponding mixed links $\widehat{B} \cup L_{1}$ and $\widehat{B} \cup L_{2}$ are isotopic in $S^{3}$ by an ambient isotopy that keeps $\widehat{B}$ pointwise fixed. See [RS72]. In terms of diagrams, the mixed link isotopy will not involve Reidemeister moves of the fixed part.

The first stage of surgery along a framed link $\widehat{B}$ is to pass from $S^{3}$ to the link complement $S^{3} \backslash \widehat{B}$. Thus, an isotopy of $L$ in $\chi\left(S^{3}, \widehat{B}\right)$ can be viewed as an isotopy in $S^{3} \backslash \widehat{B}$, but with the extra freedom for $L$ to slide across the disc that the parallel curve of a framed component of $\widehat{B}$ bounds in $\chi\left(S^{3}, \widehat{B}\right)$. This isotopy move is similar to the second move of the Kirby calculus. As noted in [LR97], the first part of the move is just isotopy in $S^{3} \backslash \widehat{B}$, so we only need to consider the essential part, where a little band of $L$ very close to the surgery component slides over the component, according to the framing and orientation conventions. We shall call this move a band move. A band move takes place in an arbitrarily thin tubular neighbourhood of the component of the surgery link, so by 'band move' we may unambiguously refer to both the move in the three-space and its projection on the plane of $\widehat{B}$. In terms of diagrams, the mixed link equivalence in $S^{3}$ includes the band moves (two types, depending on the orientation of the little band, which are related by a twist of the little band; see Figure 2). For more details the reader is referred to [LR97, Theorems 5.2 and 5.8]. 
Algebraic Markov EQUivalence For Links in THREE-MANifoldS

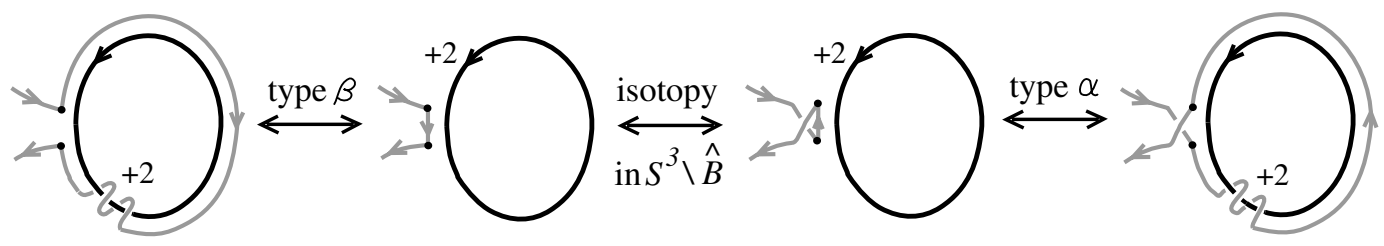

Figure 2. The two types of band moves and their relation.
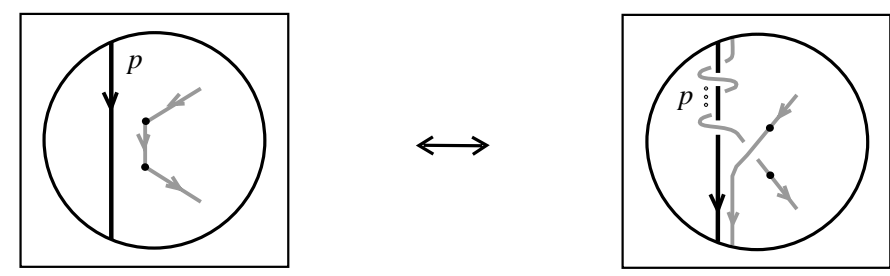

FIGURE 3. The band move for mixed braids.

Let us see now how the mixed link isotopy translates on the level of mixed braids.

Definition 1. A braid band move is a move between mixed braids, which is a band move between their closures. It starts with a little band oriented downwards, which, before sliding along a surgery strand, gets one twist positive or negative. See Figure 3. In the sequel we shall omit the word 'braid' and we shall just say 'band move'.

Definition 2 ( $L$-moves for mixed braids). Let $B \cup \beta$ be a mixed braid in $S^{3}$ and $P$ a point of an arc of the moving subbraid $\beta$, such that $P$ is not vertically aligned with any crossing or endpoint of a braid strand. Doing an $L$-move at $P$ means breaking the $\operatorname{arc}$ at $P$, bending the two resulting smaller arcs slightly apart by a small isotopy and stretching them vertically, the upper downwards and the lower upwards, and both over or under all other arcs of the diagram, so as to introduce two new corresponding strands with endpoints on the vertical line of $P$. Stretching the new strands over will give rise to an $L_{\mathrm{o}}$-move and under to an $L_{\mathrm{u}}$-move. See Figure 4.

Using a small braid isotopy, an $L$-move can be equivalently seen with a crossing (positive or negative) formed. See Figure 5.

Clearly, two mixed braids that differ by an $L$-move have isotopic closures, since the $L$-move corresponds to introducing a twist in the mixed link. $L$-moves and mixed braid isotopy generate an equivalence relation on mixed braids called $L$-equivalence. Our method of proving the one-move
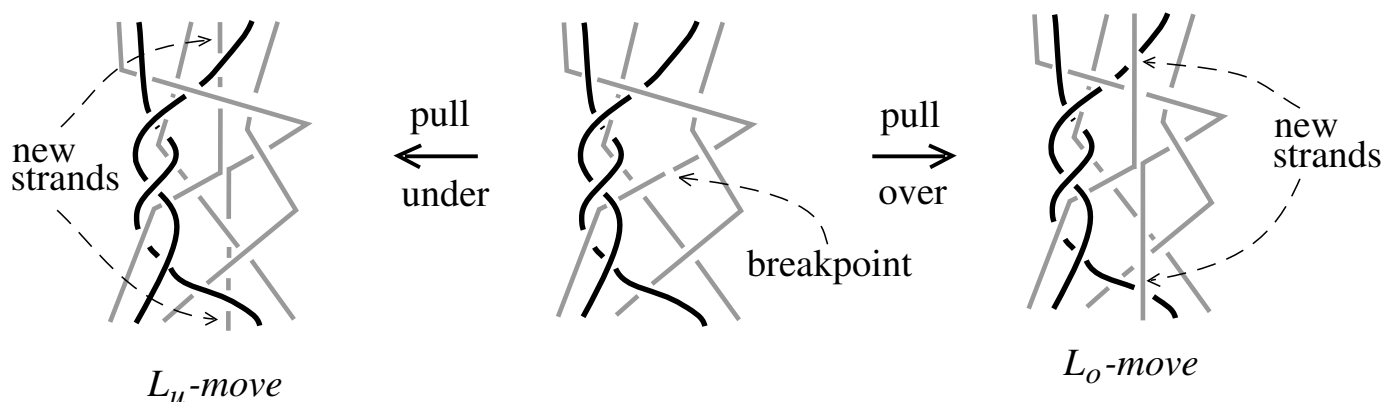

$L_{o}$-move

Figure 4. The two types of $L$-moves. 
S. LAmbropoulou And C. P. Rourke

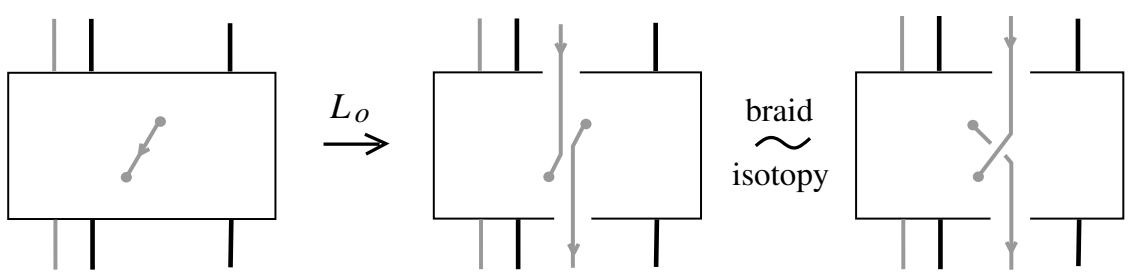

Figure 5. An $L$-move introduces a crossing.

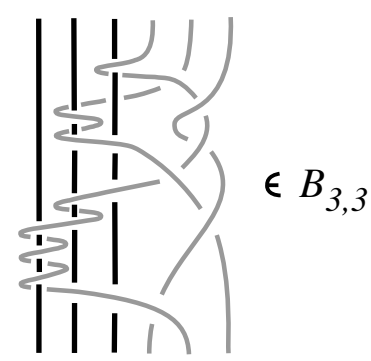

FiguRE 6. An algebraic mixed braid.

(and the classical) Markov theorem [LR97, Theorem 2.3] ensures that the arcs of the diagram that are oriented downwards do not participate in the proof. This led us to the following result [LR97, Theorems 5.5 and 5.10], which is our starting point in this paper.

Theorem 1 (Geometric Markov theorem for $V=S^{3} \backslash \widehat{B}$ or $\chi\left(S^{3}, \widehat{B}\right)$ ). Two oriented links in $S^{3} \backslash \widehat{B}$ are isotopic if and only if any two corresponding mixed braids in $S^{3}$ differ by mixed braid isotopy and a finite sequence of $L$-moves that do not touch the fixed subbraid $B$.

Moreover, if the two links lie in $\chi\left(S^{3}, \widehat{B}\right)$, the mixed braids differ by mixed braid isotopy, by $L$-moves that do not touch the fixed subbraid $B$ and by braid band moves.

The paper is concerned with the corresponding algebraic formulation.

The braid structures for links in these manifolds (as well as for links in handlebodies) have been established and studied in [Lam00]. These are either the extended braid groups $B_{m, n}$, whose elements are called algebraic mixed braids and they have the first $m$ strands forming the identity subbraid (see Figure 6 for an example), or appropriate cosets $C_{m, n}$ of these groups, that depend on the specific manifold. More precisely, $B_{m, n}$ has the presentation:

$$
B_{m, n}=\left\langle\begin{array}{l|l}
a_{1}, \ldots, a_{m}, & \begin{array}{l}
\sigma_{k} \sigma_{j}=\sigma_{j} \sigma_{k}, \quad|k-j|>1 \\
\sigma_{k} \sigma_{k+1} \sigma_{k}=\sigma_{k+1} \sigma_{k} \sigma_{k+1}, 1 \leqslant k \leqslant n-1 \\
a_{i} \sigma_{k}=\sigma_{k} a_{i}, \quad k \geqslant 2, \quad 1 \leqslant i \leqslant m \\
a_{i} \sigma_{1} a_{i} \sigma_{1}=\sigma_{1} a_{i} \sigma_{1} a_{i}, \quad 1 \leqslant i \leqslant m \\
a_{i}\left(\sigma_{1} a_{r} \sigma_{1}^{-1}\right)=\left(\sigma_{1} a_{r} \sigma_{1}^{-1}\right) a_{i}, \quad r<i
\end{array}
\end{array}\right\rangle
$$

where the generators $a_{i}$ and $\sigma_{j}$ are as illustrated in Figure 7.

The groups $B_{m, n}$ are the appropriate braid structures for studying knots and links in the complement of the $m$-unlink or a connected sum of $m$ lens spaces of type $L(p, 1)$ or a handlebody of genus $m$. For the first two cases of manifolds it is easy to formulate the analogue of the Markov theorem algebraically (see the first two examples of $\S 4$ ). In [HL02] an algebraic formulation of the Markov theorem for handlebodies was proven in terms of the groups $B_{m, n}$, one version using algebraic $L$-moves and another using Markov equivalence (cf. [HL02, Theorems 4 and 5]). In that case there 
Algebraic Markov equivalence for Links in three-manifolds

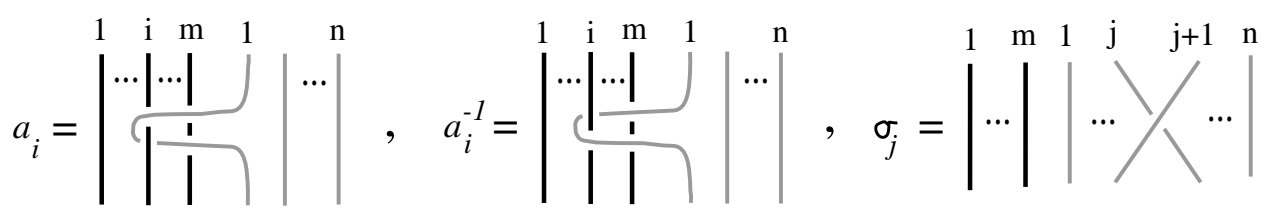

Figure 7. The 'loops' $a_{i}, a_{i}^{-1}$ and the crossings $\sigma_{j}$.

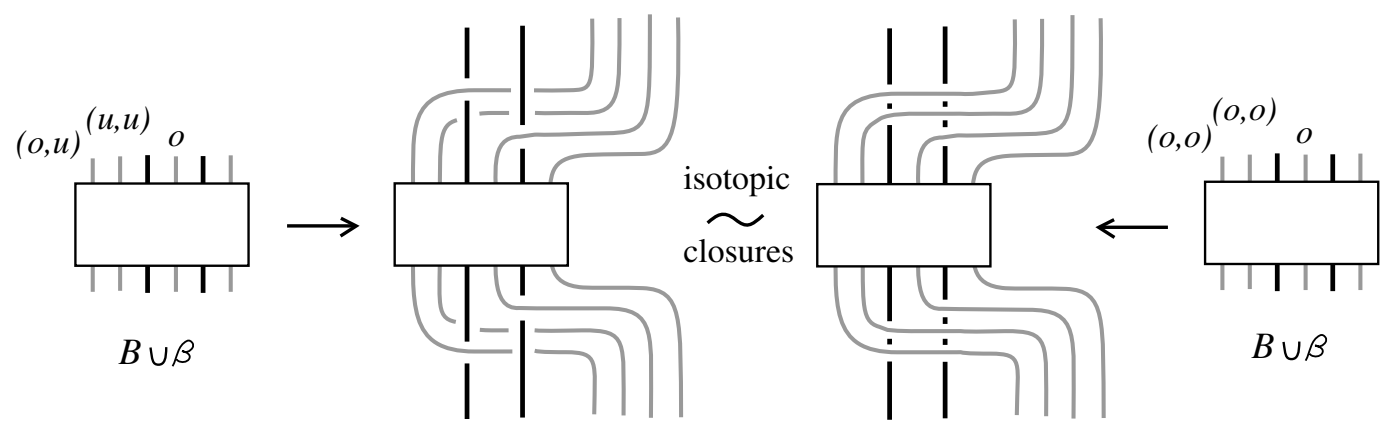

Figure 8. Parting a mixed braid - the standard parting.

was no surgery involved. The conceptual difficulty there was related to the fact that conjugations by the $a_{i}$ were not permitted.

For a generic $V=S^{3} \backslash \widehat{B}$ or $\chi\left(S^{3}, \widehat{B}\right)$ the fixed subbraid $B$ is not the identity braid. Parting a mixed braid means to separate its endpoints into two different sets, so that the resulting braids have isotopic closures. Figure 8 illustrates different partings of an abstract mixed braid. Combing a parted mixed braid means to separate the fixed subbraid from the moving part, using mixed braid isotopy. See Figure 9 for an abstract illustration. These operations are discussed in detail in $\S \S 2$ and 3. By parting and combing mixed braids, it was shown in $[$ Lam00, $\S 6$ ], that knots and links in $V$ may be represented by mixed braids in the groups $B_{m, n}$ followed by the natural embeddings of $B$ in the groups $B_{m+n}$. Hence, that the braid structures related to $V$ are the cosets of the subgroups $B_{m, n}$ in the groups $B_{m+n}(n \in \mathbb{N})$, containing the embedded fixed subbraid $B$.

\section{The main results}

The main results of this paper are Theorems 4 and 5 in $\S 3$. Theorem 4 gives the algebraic braid equivalence of combed mixed braids for knot complements and Theorem 5 gives the algebraic braid equivalence of combed mixed braids for closed three-manifolds. Our strategy for proving these theorems is the following. We first part the mixed braids and we translate the $L$-equivalence and
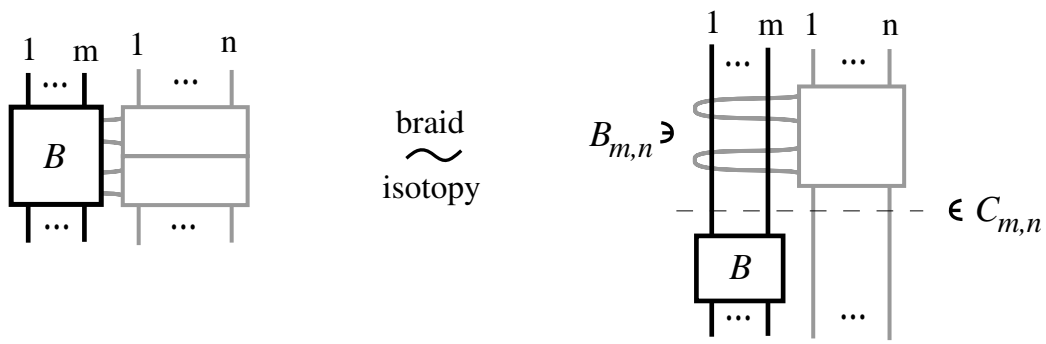

FiguRE 9. An abstract parted and combed mixed braid. 


\section{S. LAmbropoulou and C. P. Rourke}

the braid band moves of Theorem 1 to an equivalence of parted mixed braids. Here the generators of the groups $B_{m, n}$ become apparent in the equivalence. Also, the braid band moves assume a special form. These are done in $\S 2$. See Theorem 2 for link complements, and Lemma 5 and Theorem 3 for closed three-manifolds.

In $\S 3$ we comb the parted mixed braids and we translate the parted mixed braid equivalence to an equivalence of algebraic mixed braids. For both link complements and closed three-manifolds, Markov move and Markov conjugation remain equivalence moves between combed and algebraic mixed braids. However, in place of the conjugation by a 'loop' $a_{i}$ we need to introduce the twisted conjugation, which takes into account the combing of the loop through the fixed subbraid:

$$
\beta \sim a_{i}^{\mp 1} \beta \rho_{i}^{ \pm 1}
$$

where $\rho_{i}$ is the combing of the loop $a_{i}$ through $B$, for $\beta, a_{i}, \rho_{i} \in B_{m, n}$. See Figure 19 below for illustrations.

Moreover, a parted band move after combing is the composition of an algebraic band move with the combing of the parallel strand through the surgery subbraid. An algebraic band move is a braid band move between elements of the groups $B_{m, n}$ and it has the algebraic expression

$$
\beta_{1} \beta_{2} \sim \beta_{1}^{\prime} t_{k, n}^{p_{k}} \sigma_{n}^{ \pm 1} \beta_{2}^{\prime}
$$

where $\beta_{1}, \beta_{2} \in B_{m, n}, t_{k, n}$ is a Markov conjugate of the loop $a_{k}, p_{k} \in \mathbb{Z}$ is the framing of the $k$ th surgery component of the surgery link and $\beta_{1}^{\prime}, \beta_{2}^{\prime} \in B_{m, n+1}$ are the words $\beta_{1}, \beta_{2}$, but with certain substitutions that indicate the pulling of the parallel strand to the right of the braid. See Definition 7 and Figure 20 below. Then, a combed band move has the algebraic expression

$$
\beta_{1} \beta_{2} \sim \beta_{1}^{\prime} t_{k, n}^{p_{k}} \sigma_{n}^{ \pm 1} \beta_{2}^{\prime} r_{k}
$$

where $r_{k}$ is the combing of the parted parallel strand to the $k$ th surgery strand through the surgery braid. For an illustration here see Figure 21 below.

Finally, in $\S 4$ we give explicit examples, including complements of daisy chains, the lens spaces, the complement of the Borromean rings or a closed three-manifold obtained by surgery along them. We also discuss the case where the surgery braid is not a pure braid (Lemma 9) and we present as an example the complement of a trefoil or a manifold obtained by surgery along it.

This paper is a sequel to [LR97, Lam00, HL02]. It sets out the necessary algebraic formalism for constructing knot invariants in three-manifolds using braid machinery, for example via constructing Markov traces on appropriate algebras, quotients of the group algebras of the braid groups $B_{m, n}$. (See [Jon87] for the classical case of links in $S^{3}$ ). In the case $m=1, B_{1, n}$ is the Artin group of type $\mathcal{B}$. See, for example, [Lam99] and references therein for the construction of the analogues of the two-variable Jones polynomial (homflypt) for links in the solid torus. The case of $L(p, 1)$ is being studied by the first author with J. H. Przytycki. Theorem 5 gives very good control over the band moves of links in closed three-manifolds, and this is very useful for the study of skein modules of three-manifolds. For skein modules of three-manifolds see, for example, [Prz06] and references therein.

A final comment is now due. In our set-up the manifold is represented in $S^{3}$ by a fixed link. In the case of a closed three-manifold the surgery link is not unique up to isotopy. In fact, it may be altered via the Kirby calculus [Kir78]. Then the corresponding surgery braids are equivalent under moves described in [KS92]. One could then consider combining the mixed braid equivalence given in the present paper with the braid equivalence of [KS92]. This will be the subject of a subsequent paper. 


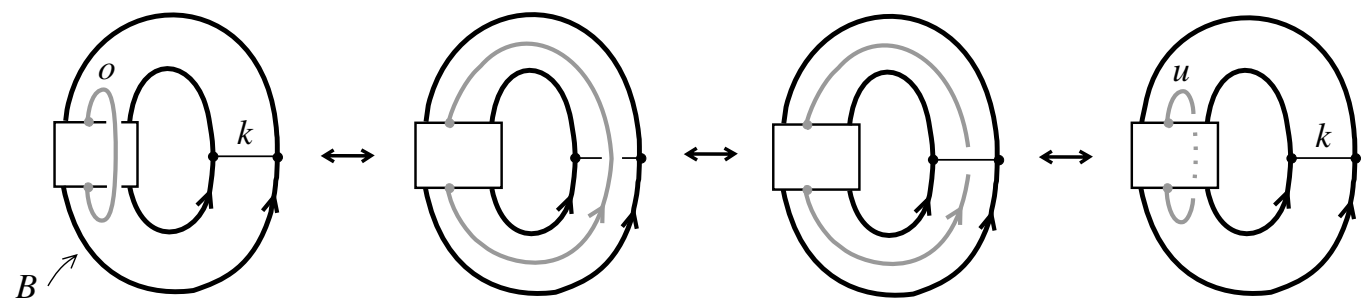

Figure 10. Crossing the arc $k$.

\section{Markov equivalence for parted mixed braids}

This section is an intermediate step towards the algebrization of Theorem 1 . Here the mixed braids resulting from the mixed links have all, say $m$, strands of the fixed part $B$ occupying the first $m$ positions of the mixed braid.

Definition 3. A parted mixed braid is a mixed braid $B \cup \beta$ on $m+n$ strands, such that the first $m$ endpoints are those of the subbraid $B$ and the last $n$ endpoints are those of $\beta$. Parted mixed braids are denoted in the same way as mixed braids. We number their fixed strands from 1 up to $m$ and their moving strands from 1 up to $n$. (See the left-hand side of Figure 14 below for an abstract illustration.)

Lemma 1. Every mixed braid may be represented by a parted mixed braid with isotopic closure (cf. [Lam00, § 6], compare with [HL02, Lemma 1]).

Proof. Indeed, let $B \cup \beta$ be a mixed braid. To see this we simply attach arbitrarily arrays of labels 'o' or ' $\mathrm{u}$ ' to corresponding pairs of endpoints of the moving subbraid $\beta$, with as many entries as the number of fixed strands on their right, and we pull the strands of corresponding endpoints to the right, over or under each strand of $B$ that lies on their right, according to the label in the array of the pulled strands. We start from the rightmost pair respecting the position of the endpoints. View the first two illustrations of Figure 8 for the parting of an abstract mixed braid. Obviously, the closures of the initial and of the parted mixed braid are isotopic (they differ by planar isotopy and by mixed Reidemeister II moves).

It follows that two different partings of a mixed braid give rise, upon closure, to isotopic mixed links. For this reason we could fix the pulling of the moving strands during the parting process to be always over or always under each strand of $B$ that lies on its right. If the pulling is always over we shall refer to it as the standard parting. See the two illustrations on the right of Figure 8.

Pulling a moving strand 'under' a fixed strand instead of 'over' it simply corresponds to the fact that the closure of the moving strand crosses a hypothetical closing arc $k$ of the fixed subbraid $B$, and this is an allowed isotopy move in the manifolds considered here. See Figure 10. (We note that this is not true in the case of handlebodies and this is the reason why conjugation by the generators $a_{i}$ is not permitted; see [HL02] for a detailed analysis.)

Lemma 2 below gives the relation of an arbitrary parting with the corresponding standard parting, and it is very instructive, as it brings the 'loops' $a_{i}$ into the parted braid equivalence. Note that the elementary algebraic mixed braids $a_{i}$ and their inverses, together with the crossings $\sigma_{j}$ (all defined in Figure 7) are clearly the geometric generators of the moving part of a parted mixed braid.

Now let $C_{m, n}$ denote the set of parted mixed braids on $n$ moving strands related to $V=S^{3} \backslash \widehat{B}$ or $\chi\left(S^{3}, \widehat{B}\right)$. By adding an extra moving strand on the right of a parted mixed braid on $n$ moving 


\section{S. Lambropoulou and C. P. Rourke}

strands, $C_{m, n}$ embeds naturally into $C_{m, n+1}$. Let $C_{m, \infty}:=\cup_{n=1}^{\infty} C_{m, n}$ denote the disjoint union of all sets $C_{m, n}$. We define below some moves in $C_{m, \infty}$.

\section{DEFINITION 4.}

(1) Loop conjugation of a parted mixed braid in $C_{m, n}$ is its concatenation from above by a loop $a_{i}$ (or by $a_{i}^{-1}$ ) and from below by $a_{i}^{-1}$ (respectively $a_{i}$ ).

(2) Markov conjugation of a parted mixed braid in $C_{m, n}$ is its concatenation from above by a crossings $\sigma_{j}$ (or by $\sigma_{j}^{-1}$ ) and from below by $\sigma_{j}^{-1}$ (respectively $\sigma_{j}$ ).

(3) A parted $L$-move is defined to be an $L$-move between parted mixed braids. (See the left-hand illustration of Figure 15 below.)

(4) An $M$-move is the insertion of a crossing $\sigma_{n}^{ \pm 1}$ at the right-hand side of a parted mixed braid on $n$ moving strands. Undoing an $M$-move is the reverse operation. See Figure 13 below.

Lemma 2. Consider a mixed braid on $m+n$ strands and an arbitrary parting of it in $C_{m, n}$. Then, up to Markov conjugation, this parting differs from its corresponding standard parting by a finite sequence of loop conjugations.

Proof. By an inductive argument we may assume that all moving strands from the first up to the $(j-1)$ th are pulled 'over' all the fixed strands that lie on their right. Consider now the $j$ th moving strand. Upon parting, this lands on the $j$ th position of the moving part of the resulting parted mixed braid. See Figure 11. By a mixed braid isotopy we bring the $j$ th moving strand on top of the other moving strands. Note that the braid isotopy is independent of the parting labels attached to the $j$ th moving strand. Then by Markov conjugation by the word $\left(\sigma_{1} \ldots \sigma_{j-1}\right)$ we bring the $j$ th moving strand to the first position of the moving subbraid. See the top row of Figure 11.

Now let the parting label of the original $j$ th moving strand for the $i$ th fixed strand be 'under'. By a second inductive argument we may assume that the parting labels of the original $j$ th strand are all 'over' for the $(i+1)$ th up to the $m$ th fixed strand. Then, conjugation by $a_{i}$ changes the label 'under' to 'over'. By applying once more mixed braid isotopy and Markov conjugation by the word $\left(\sigma_{j-1}^{-1} \ldots \sigma_{1}^{-1}\right)$ we obtain a parted mixed braid identical to the initial one except for the place of the one crossing in question, which is switched. See the second row of Figure 11. Continuing backwards with the remaining parting labels of the $j$ th moving strand we change them all in this manner to 'over', and this ends the proof.

Remark 1. It follows from the proof of Lemma 2 that changing a parting label from 'under' to 'over' corresponds in $C_{m, \infty}$ to conjugation by some $a_{i}$.

Lemma 3. A mixed braid with an L-move performed can be parted to a parted mixed braid with a parted L-move performed. (Compare with [HL02, Lemma 2].)

Proof. If the $L$-move is an $L_{\mathrm{o}}$-move, we part its strands by pulling them to the right and over all other strands in between. Then the crossing of the $L$-move slides over to the right by a braid isotopy. See Figure 12. The case of an $L_{\mathrm{u}}$-move is analogous: here we pull the two strands under the fixed strands in between.

Lemma 4. Markov conjugation and $M$-moves can be realized by a sequence of parted $L$-moves. Conversely, a parted L-move is a composition of an $M$-move and Markov conjugation.

Proof. It is clear that an $M$-move is a special case of a parted $L$-move. The one-move Markov theorem in $S^{3}$ implies that Markov conjugation in $S^{3}$ can be realized by a sequence of $L$-moves (cf. [LR97, § 4.1]). The same arguments apply to both link complements and closed three-manifolds. However, we would like to give a second direct proof of Lemma 4, which is an adaptation for 

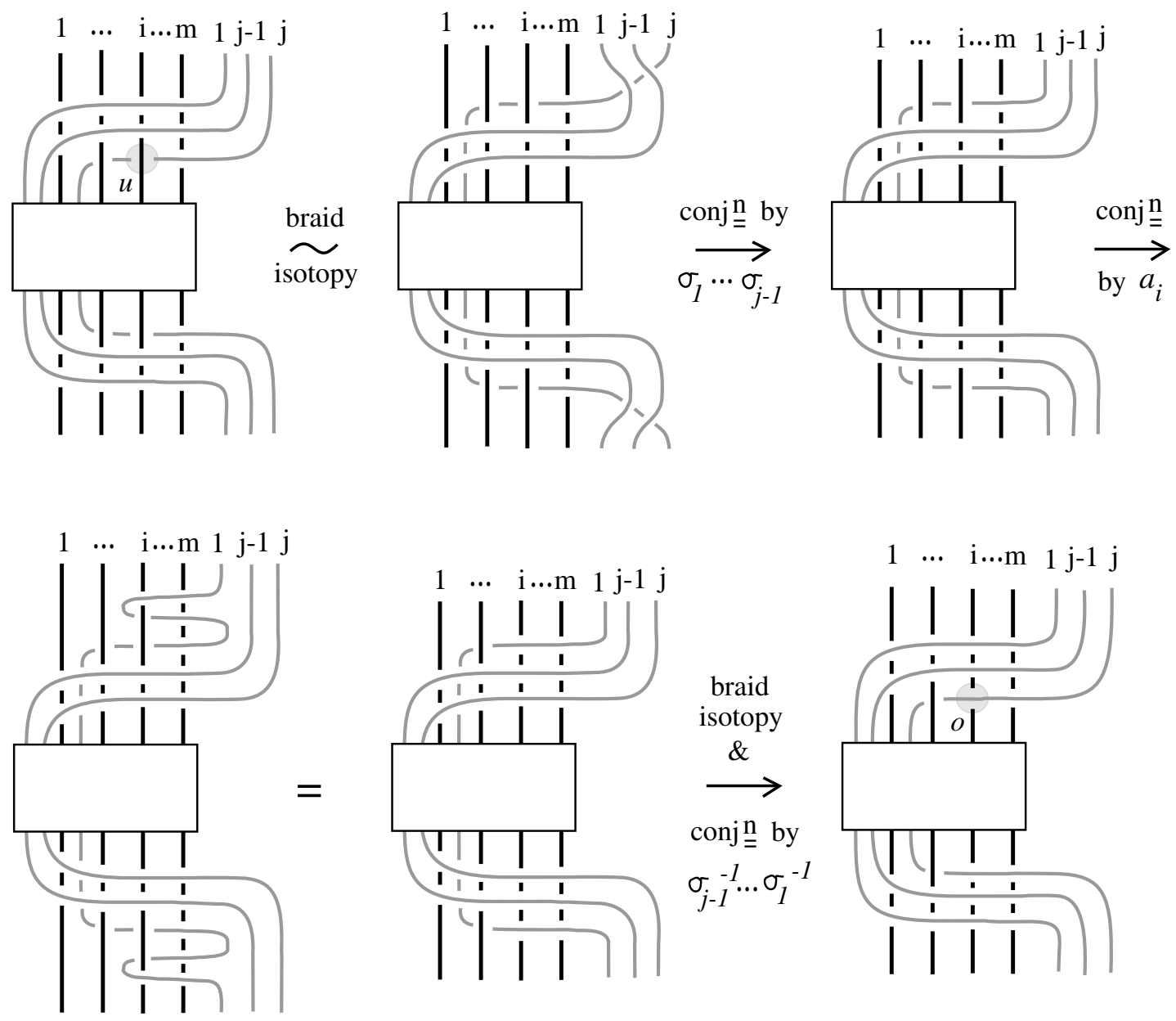

Figure 11. Change of parting labels $\longleftrightarrow$ conjugation by $a_{i}$.

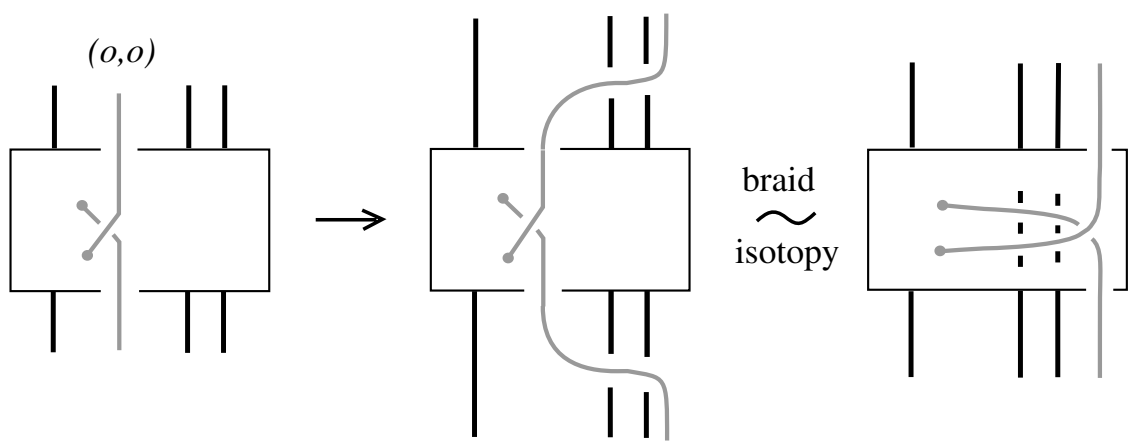

FiguRE 12. Sliding an $L_{\mathrm{o}}$-move to the right. 


\section{S. Lambropoulou and C. P. Rourke}
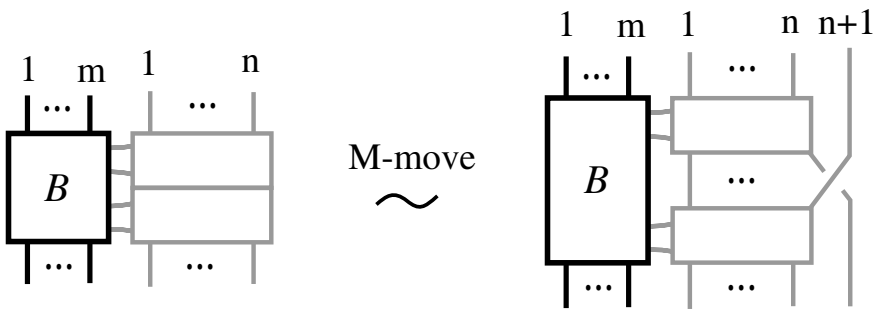

Figure 13. The $M$-move.

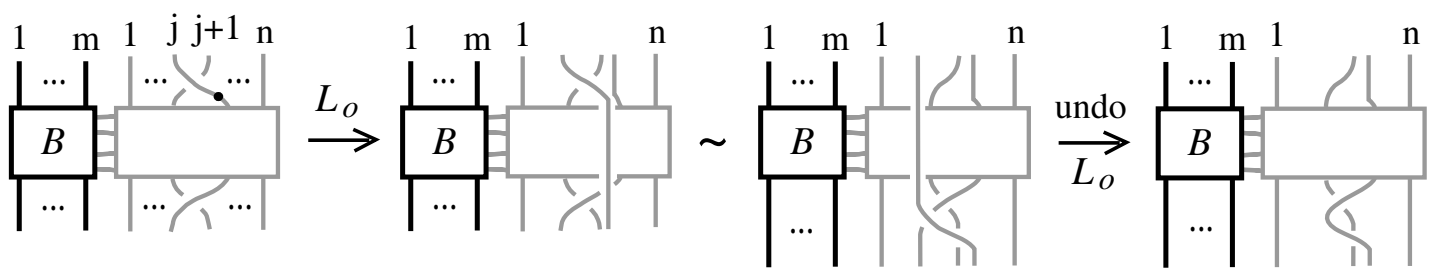

Figure 14. Conjugation by $\sigma_{j}^{-1}$ is a composition of $L$-moves.

the case of knot complements and closed three-manifolds of a direct proof for the classical case of $S^{3}$, given by Häring-Oldenburg. In Figure 14 we start with a parted mixed braid conjugated by $\sigma_{j}$. After performing an appropriate parted $L_{\mathrm{O}}$-move, braid isotopy and undoing another parted $L_{\mathrm{O}^{-}}$ move, we end up with the original mixed braid free of conjugation by the $\sigma_{j}$. Conversely, as it becomes clear from Figure 15, a parted $L$-move is a composition of an $M$-move and Markov conjugation.

We are now in a position to state two versions of the analogue of the Markov theorem for parted mixed braids in $S^{3} \backslash \widehat{B}$.

Theorem 2 (Parted version of Markov theorem for $V=S^{3} \backslash \widehat{B}$ ). Two oriented links in $S^{3} \backslash \widehat{B}$ are isotopic if and only if any two corresponding parted mixed braids in $C_{m, \infty}$ differ by a finite sequence of parted $L$-moves and loop conjugations.

Equivalently, two oriented links in $S^{3} \backslash \widehat{B}$ are isotopic if and only if any two corresponding parted mixed braids in $C_{m, \infty}$ differ by a finite sequence of $M$-moves, Markov conjugations and loop conjugations.

Proof. This follows immediately from Theorem 1 for $V=S^{3} \backslash \widehat{B}$ and from Lemmas 2, 3 and 4 .
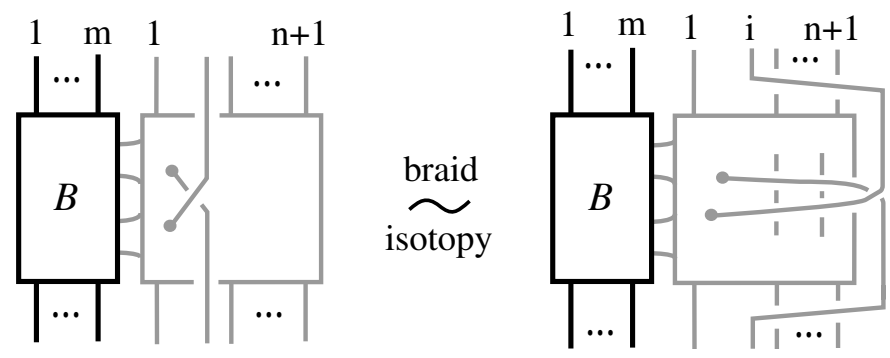

FiguRE 15. Parted $L$-move $\longleftrightarrow M$-move and Markov conjugation. 

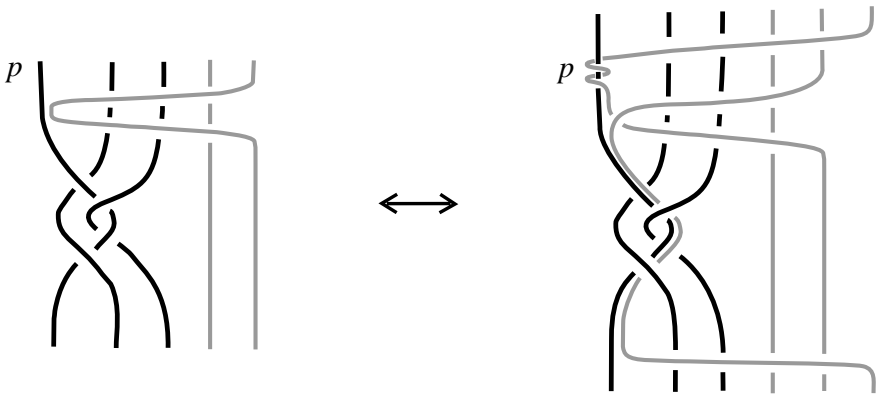

Figure 16. A parted band move.

We would like to extend Theorem 2 to parted mixed braids in closed three-manifolds. Lemma 5 below sharpens the band moves of Theorem 1 for $\chi\left(S^{3}, \widehat{B}\right)$ and it shows the effect of parting on band moves.

Definition 5. A parted band move is defined to be a band move between parted mixed braids, such that: it takes place at the top part of the braid (before any surgery braid crossings are encountered) and the little band starts from the last strand of the moving subbraid and it moves over each moving strand and each component of the surgery braid, until it reaches from the right the specific component. After the band move is performed we apply to the resulting mixed braid the standard parting bringing the new strands over to the last position of the moving part.

See Figure 16 for an example of a parted positive band move, where the moving part has been simplified to the identity braid.

Lemma 5. A band move may be always assumed, up to L-equivalence, to take place at the top part of a mixed braid and on the right of the specific surgery strand.

Moreover, performing a band move on a parted mixed braid and then parting, the result is equivalent, up to L-moves and loop conjugation, to performing a parted band move.

Proof. In a mixed braid $B \cup \beta_{1}$ consider a little band that has approached a specific surgery strand of $B$ from the right and is about to perform a band move. Pull the little band up to the top along the surgery strand. See illustrations 1 and 3 of Figure 17. Then perform braiding to obtain a mixed braid $B \cup \beta_{2}$. This is $L$-equivalent to $B \cup \beta_{1}$. Note that the edge arc of the little band is still there in $B \cup \beta_{2}$, because it is a down-arc. Now, using this arc, perform a top band move in $B \cup \beta_{2}$ and call the resulting mixed braid $B \cup \alpha_{2}$. See illustration 4 of Figure 17. Let also $B \cup \alpha_{1}$ be the mixed braid obtained after performing the band move in the first mixed braid $B \cup \beta_{1}$. See illustration 2 of Figure 17, but consider only the braid between the dotted lines. Then $B \cup \alpha_{1}$ differs from $B \cup \alpha_{2}$ by exactly the same sequence of $L$-moves, $L_{1}, \ldots, L_{k}$ say, that separate $B \cup \beta_{1}$ and $B \cup \beta_{2}$, since the isotopies separating the corresponding closures are identical. Compare the corresponding diagrams of Figure 17. Thus, we have shown that

$$
\begin{gathered}
B \cup \beta_{1} \stackrel{\text { general band move }}{\sim} B \cup \alpha_{1} \\
\Longleftrightarrow \\
B \cup \beta_{1} \stackrel{L_{1}, \ldots, L_{k}}{\sim} B \cup \beta_{2} \stackrel{\text { top band move }}{\sim} B \cup \alpha_{2} \stackrel{L_{1}{ }^{-1}, \ldots, L_{k}{ }^{-1}}{\sim} B \cup \alpha_{1} .
\end{gathered}
$$

The first statement of the lemma is proved. Consider now the same setting as above, but with $B \cup \beta_{1}$ being a parted mixed braid. Perform on it a band move and part the resulting new mixed braid by pulling the two new strands over all strands in between to the last position of the moving part. See illustration 2 of Figure 17, where the parting is now included. At the same time pull the 

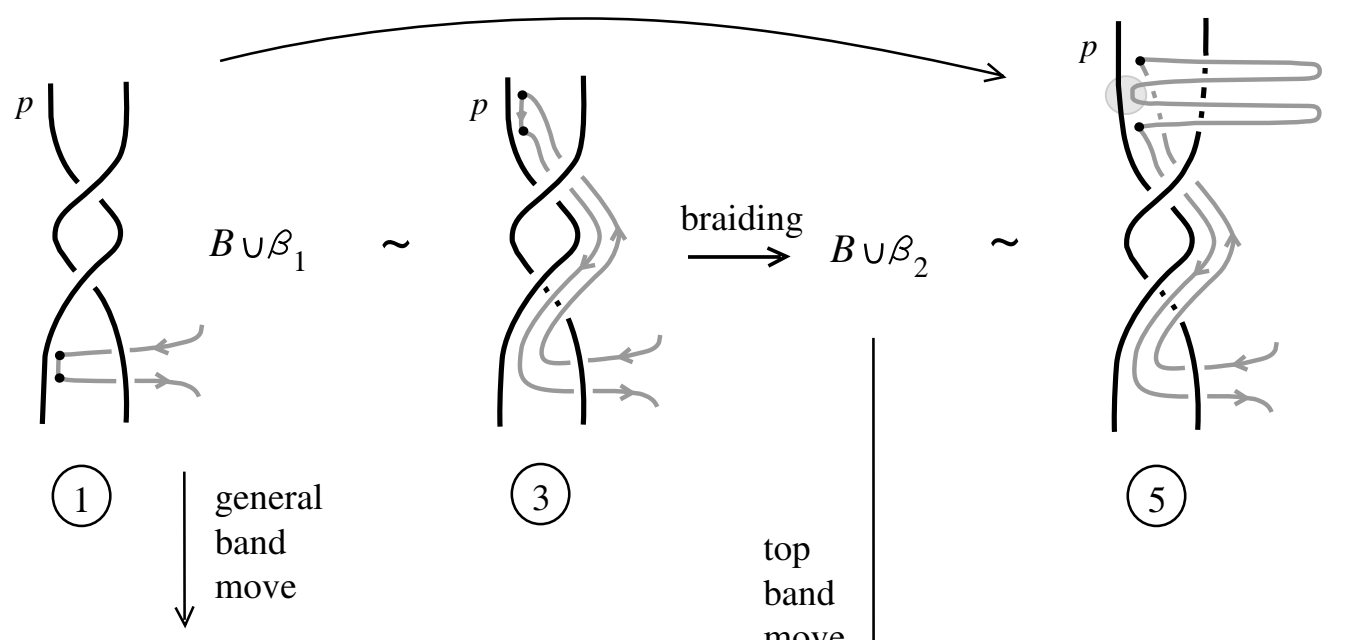

(3)
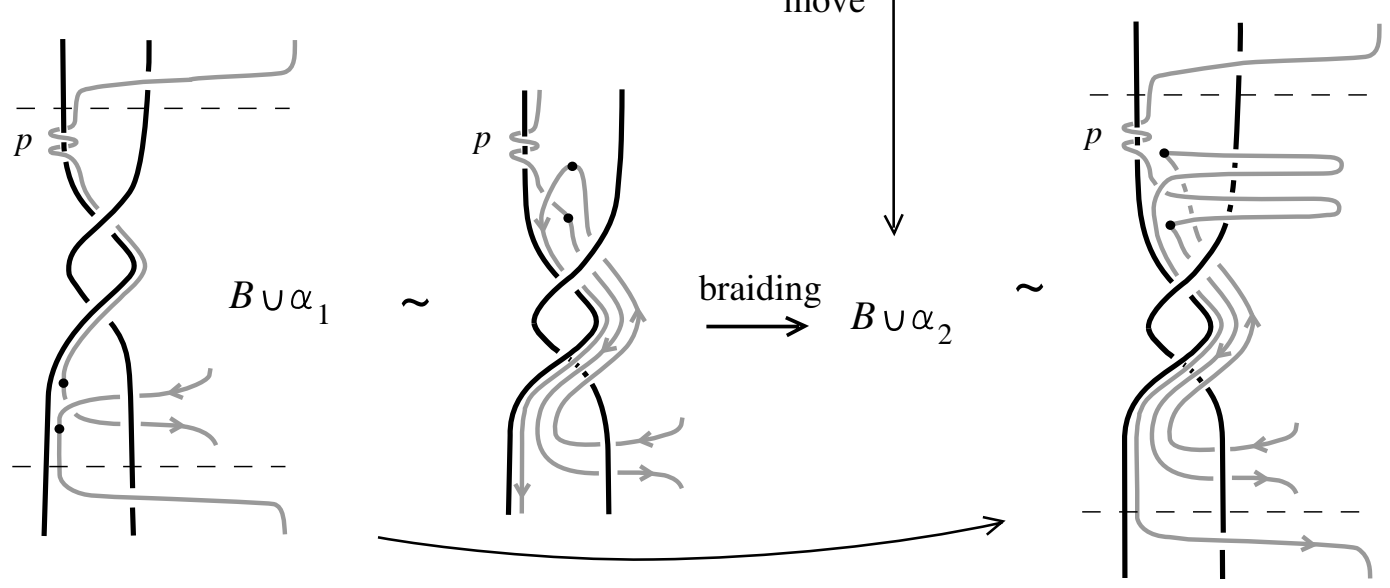

(2)

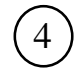

(6)

FiguRE 17. The proof of Lemma 5.

little band of $B \cup \beta_{1}$ up to the top and then horizontally to the right, over all strands in between, until it reaches the last position of the moving part. Then do a similar pull-back to the left up to the specified surgery strand. See illustration 5 of Figure 17. Now perform a parted band move at the place marked with a shaded disc. See illustration 6 of Figure 17. As above, this last mixed braid operation does not create any new up-arcs and it does not interfere with the band move. Finally, part by the standard parting the new strands created by braiding the up-arcs from the pulling along the surgery strand, and part the new strands created from the parted band move last, by pulling them over all strands in between to the last position of the moving part. Clearly, the two pairs (related to $(1,5)$ and $(2,6)$ of Figure 17) of parted mixed braids involved differ by the same parted $L$-moves together with loop conjugation that comes from the parting.

Further note that, by braid isotopy and loop conjugation, the $p$ twists of a general band move may take place anywhere along the surgery strand, so just as well at the top, as in Definition 5 of a parted band move. Thus, we showed that a band move on a parted mixed braid can be accomplished with a parted band move, up to $L$-moves and loop conjugation.

Finally, if the little band lies on the left of the surgery component we pull it horizontally over the surgery strand and to the right and then we pull it slightly back to the left, so that it approaches the surgery strand from the right. Up to here it is only braid isotopy. We now perform a band move 


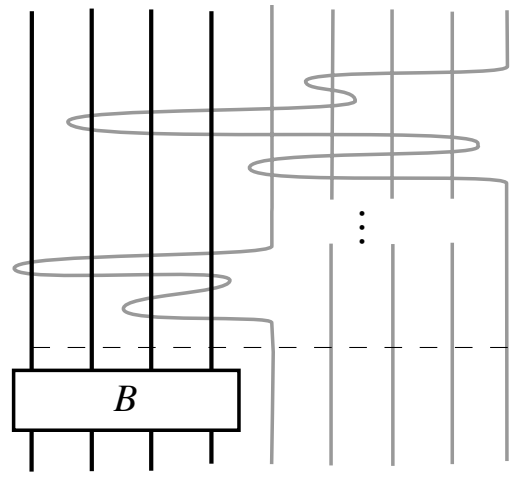

FiguRE 18. Artin's combing separates the fixed part from the moving part.

before and a band move after and we notice that the two final mixed braids differ by conjugating a half twist of the framing. In any case, after parting the two final mixed braids are the same.

Then Theorem 1 for $\chi\left(S^{3}, \widehat{B}\right)$ and Lemma 5 extend Theorem 2 to the following.

Theorem 3 (Parted version of Markov theorem for $\chi\left(S^{3}, \widehat{B}\right)$ ). Two oriented links in $\chi\left(S^{3}, \widehat{B}\right)$ are isotopic if and only if any two corresponding parted mixed braids in $C_{m, \infty}$ differ by a finite sequence of parted L-moves, loop conjugations and parted band moves.

Equivalently, two oriented links in $\chi\left(S^{3}, \widehat{B}\right)$ are isotopic if and only if any two corresponding parted mixed braids in $C_{m, \infty}$ differ by a finite sequence of $M$-moves, Markov conjugations, loop conjugations and parted band moves.

\section{Markov equivalence for combed and algebraic mixed braids}

In this section we explain the combing of parted mixed braids and we translate the equivalence of Theorems 2 and 3 to equivalence of algebraic mixed braids.

Let $V=S^{3} \backslash \widehat{B}$ or $\chi\left(S^{3}, \widehat{B}\right)$. Unless $V$ is the complement of the $m$-unlink or a connected sum of $m$ lens spaces of type $L(p, 1)$, where the fixed subbraid $B$ is the identity braid on $m$ strands, concatenating two elements of $C_{m, n}$ is not a closed operation, since it alters the braid description of the manifold. So, the set $C_{m, n}$ of parted mixed braids is not a subgroup of $B_{m+n}$. Yet, as shown in $[$ Lam00, $\S 6]$, using Artin's combing for pure braids, the moving part of a parted mixed braid can be combed away from the fixed subbraid, so that this latter remains free of mixed linking at the bottom of the parted mixed braid. Thus, the parted mixed braid splits into the concatenation of two parted mixed braids: the 'algebraic' part at the top, which has as fixed subbraid the identity braid on $m$ strands and the 'coset' part at the bottom consisting of the fixed braid $B$ embedded naturally in $B_{m+n}$. See Figure 18 for an abstract illustration. The result will be called a combed mixed braid.

Recall that the algebraic part of a combed mixed braid is called algebraic mixed braid and it is an element of $B_{m, n}$. (Recall Figure 6 for an example.) The set $B_{m, n}$ of all algebraic mixed braids on $m$ fixed strands and $n$ moving strands is closed under the usual concatenation and with respect to inverses. Thus, it is a subgroup of $B_{m+n}$. The set $C_{m, n}$ of combed mixed braids is a coset of $B_{m, n}$ in $B_{m+n}$ (cf. [Lam00, Proposition 1]). Thus, for a fixed manifold $V$, an element in $B_{m, n}$ represents unambiguously an element in $C_{m, n}$, hence an oriented link in $V$. The braid group $B_{m, n}$ embeds naturally into the group $B_{m, n+1}$ and we shall denote by $B_{m, \infty}:=\bigcup_{n=1}^{\infty} B_{m, n}$ the disjoint union of all braid groups $B_{m, n}$. 


\section{S. LAmbropoulou and C. P. Rourke}

We would like to restate the Markov equivalence in Theorems 2 and 3 for parted mixed braids in terms of their corresponding algebraic mixed braids after combing. For this we need to understand exactly how the combing is done and how it affects the parted braid equivalence moves.

Note that, if we regard a parted mixed braid as an element of the classical braid group $B_{m+n}$, then the crossings $\sigma_{j}$ of the moving part commute with the crossings of the fixed part, so they are not affected by combing. More precisely, if $\Sigma_{k}$ denotes the crossing between the $k$ th and the $(k+1)$ th strand of the fixed subbraid, then for all $j=1,2, \ldots, n-1$ and $k=1,2, \ldots, m-1$ we have the relations

$$
\Sigma_{k} \sigma_{j}=\sigma_{j} \Sigma_{k}
$$

Thus, the only generating elements of the moving part that are affected by the combing are the loops $a_{i}$. In Lemma 6 below we give formuli for the effect of combing on the $a_{i}$.

Lemma 6. The crossings $\Sigma_{k}$, for $k=1, \ldots, m-1$, and the loops $a_{i}$, for $i=1, \ldots, m$, satisfy the following 'combing' relations:

- $\Sigma_{k} a_{k}^{ \pm 1}=a_{k+1}^{ \pm 1} \Sigma_{k}$;

- $\Sigma_{k} a_{k+1}^{ \pm 1}=a_{k+1}^{-1} a_{k}^{ \pm 1} a_{k+1} \Sigma_{k}$;

- $\Sigma_{k} a_{i}^{ \pm 1}=a_{i}^{ \pm 1} \Sigma_{k}$, if $i \neq k, k+1$;

- $\Sigma_{k}^{-1} a_{k}^{ \pm 1}=a_{k} a_{k+1}^{ \pm 1} a_{k}^{-1} \Sigma_{k}^{-1}$;

- $\Sigma_{k}^{-1} a_{k+1}^{ \pm 1}=a_{k}^{ \pm 1} \Sigma_{k}^{-1}$;

- $\Sigma_{k}^{-1} a_{i}^{ \pm 1}=a_{i}^{ \pm 1} \Sigma_{k}^{-1}$, if $i \neq k, k+1$.

Moreover, since $B$ is assumed to be a pure braid for $V=\chi\left(S^{3}, \widehat{B}\right)$, it is useful to give the 'combing' relations between the crossings $\Sigma_{k}^{2}$ and the loops $a_{i}$. Indeed we have:

- $\Sigma_{k}^{2} a_{k}^{ \pm 1}=a_{k+1}^{-1} a_{k}^{ \pm 1} a_{k+1} \Sigma_{k}^{2}$;

- $\Sigma_{k}^{2} a_{k+1}^{ \pm 1}=a_{k+1}^{-1} a_{k}^{-1} a_{k+1}^{ \pm 1} a_{k} a_{k+1} \Sigma_{k}^{2}$;

- $\Sigma_{k}^{2} a_{i}^{ \pm 1}=a_{i}^{ \pm 1} \Sigma_{k}^{2}$, if $i \neq k, k+1$;

- $\Sigma_{k}^{-2} a_{k}^{ \pm 1}=a_{k} a_{k+1} a_{k}^{ \pm 1} a_{k+1}^{-1} a_{k}^{-1} \Sigma_{k}^{-2}$;

- $\Sigma_{k}^{-2} a_{k+1}^{ \pm 1}=a_{k} a_{k+1}^{ \pm 1} a_{k}^{-1} \Sigma_{k}^{-2}$;

- $\Sigma_{k}^{-2} a_{i}^{ \pm 1}=a_{i}^{ \pm 1} \Sigma_{k}^{-2}$, if $i \neq k, k+1$.

Proof. We illustrate in Figure 19 the first three principal relations for $\Sigma_{1}$. For arbitrary $\Sigma_{k}$ the proof is obviously analogous. The relations for the crossings $\Sigma_{k}^{-1}$ and for $\Sigma_{k}^{2}$ follow easily from those for $\Sigma_{k}$.

In $B_{m, \infty}$ we now define the following moves.

\section{DEFINITION 6.}

(1) Twisted loop conjugation is defined to be a combed loop conjugation and it has the algebraic expressions

$$
\beta \sim a_{i}^{\mp 1} \beta \rho_{i}^{ \pm 1}
$$

for $\beta, a_{i}, \rho_{i} \in B_{m, n}$, where $\rho_{i}$ is the combing of the loop $a_{i}$ through the fixed braid $B$. (Note that the combing of the loop $a_{i}^{-1}$ through the fixed braid $B$ is $\rho_{i}{ }^{-1}$.)

(2) Algebraic Markov conjugation is Markov conjugation between elements of $B_{m, \infty}$ and it has the algebraic expression

where $\alpha, \sigma_{j} \in B_{m, n}$.

$$
\alpha \sim \sigma_{j}^{ \pm 1} \alpha \sigma_{j}^{\mp 1}
$$


Algebraic Markov equivalence for links in three-manifolds
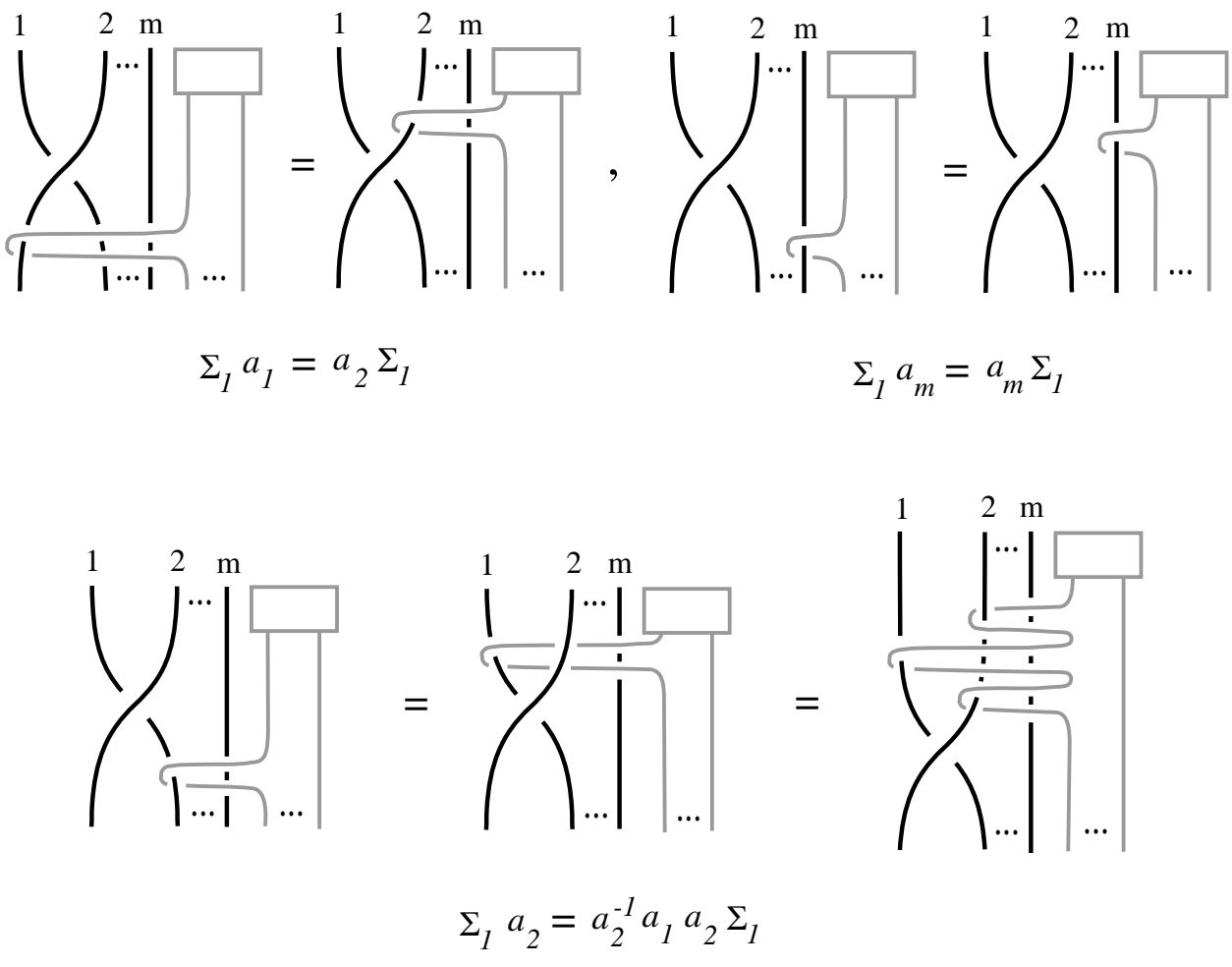

Figure 19. The relations between $\Sigma_{1}$ and the $a_{i}$.

(3) An algebraic $L$-move is a parted $L$-move between elements of $B_{m, \infty}$. From Figure 15 one can easily derive the following algebraic expressions for algebraic $L_{\mathrm{o}}$-moves and algebraic $L_{\mathrm{u}}$-moves, respectively.

$$
\begin{gathered}
\alpha=\alpha_{1} \alpha_{2} \sim \sigma_{i}^{-1} \ldots \sigma_{n}^{-1} \alpha_{1} \sigma_{i-1}^{-1} \ldots \sigma_{n-1}^{-1} \sigma_{n}^{ \pm 1} \sigma_{n-1} \ldots \sigma_{i} \alpha_{2} \sigma_{n} \ldots \sigma_{i} \\
\alpha=\alpha_{1} \alpha_{2} \sim \sigma_{i} \ldots \sigma_{n} \alpha_{1} \sigma_{i-1} \ldots \sigma_{n-1} \sigma_{n}^{ \pm 1} \sigma_{n-1}^{-1} \ldots \sigma_{i}^{-1} \widetilde{\alpha_{2}} \sigma_{n}^{-1} \ldots \sigma_{i}^{-1}
\end{gathered}
$$

where $\alpha_{1}, \alpha_{2} \in B_{m, n}$.

(4) An algebraic $M$-move is an $M$-move between elements of $B_{m, \infty}$ and it has the algebraic expression

where $\alpha_{1}, \alpha_{2} \in B_{m, n}$.

$$
\alpha_{1} \alpha_{2} \sim \alpha_{1} \sigma_{n}^{ \pm 1} \alpha_{2}
$$

Lemma 7. Two parted mixed braids that differ by Markov conjugation by some $\sigma_{j}$, respectively by an $M$-move, respectively by a parted $L$-move, after combing they give rise to algebraic mixed braids that differ by algebraic Markov conjugation by the $\sigma_{j}$, respectively by an algebraic $M$-move, respectively by an algebraic $L$-move.

Proof. As observed earlier, the crossings of the moving part commute with the crossings of the fixed part. Thus, Markov conjugation, the $M$-moves and the parted $L$-moves all commute with combing. Moreover, the two parted mixed braids are otherwise identical, so they are both combed in exactly the same manner. Therefore, after combing, the combed mixed braids as well as their corresponding algebraic mixed braids will just differ by algebraic Markov conjugation, respectively an algebraic $M$-move, respectively an algebraic $L$-move.

We are now in a position to restate Theorem 2 in terms of algebraic mixed braids. 


\section{S. LAmbropoulou and C. P. Rourke}

Theorem 4 (Algebraic Markov theorem for $S^{3} \backslash \widehat{B}$ ). Two oriented links in $S^{3} \backslash \widehat{B}$ are isotopic if and only if any two corresponding algebraic mixed braid representatives in $B_{m, \infty}$ differ by a finite sequence of the following moves:

(1) algebraic $M$-moves: $\alpha_{1} \alpha_{2} \sim \alpha_{1} \sigma_{n}^{ \pm 1} \alpha_{2}$, for $\alpha_{1}, \alpha_{2} \in B_{m, n}$;

(2) algebraic Markov conjugation: $\alpha \sim \sigma_{j}^{ \pm 1} \alpha \sigma_{j}^{\mp 1}$, for $\alpha, \sigma_{j} \in B_{m, n}$;

(3) twisted loop conjugation: $\beta \sim a_{i}^{\mp 1} \beta \rho_{i}^{ \pm 1}$, for $\beta, a_{i}, \rho_{i} \in B_{m, n}$, where $\rho_{i}$ is the combing of the loop $a_{i}$ through $B$;

or, equivalently, by a finite sequence of the following moves:

$\left(1^{\prime}\right)$ algebraic L-moves (see the algebraic expressions in Definition 6);

$\left(2^{\prime}\right)$ twisted loop conjugation.

Proof. By Lemma 7, $M$-moves and Markov conjugation get combed to algebraic $M$-moves and algebraic Markov conjugation. Thus, by Theorem 2, we only have to observe that conjugating a parted mixed braid by a loop $a_{i}$ induces after combing the twisted conjugation on the level of the corresponding algebraic braids. Lemma 6 explains how to efficiently perform the combing of the loops $a_{i}^{ \pm 1}$.

In order to extend Theorem 4 to mixed braids in $\chi\left(S^{3}, \widehat{B}\right)$ we need to understand how a parted band move is combed through the surgery braid $B$ and to give algebraic expressions for parted band moves between algebraic mixed braids.

Definition 7. An algebraic band move is defined to be a parted band move between elements of $B_{m, \infty}$. See Figure 20 for an abstract example. Setting

$$
\lambda_{n-1}:=\sigma_{n-1} \cdots \sigma_{1} \quad \text { and } \quad t_{k, n}:=\sigma_{n} \cdots \sigma_{1} a_{k} \sigma_{1}^{-1} \cdots \sigma_{n}^{-1},
$$

an algebraic band move has the following algebraic expression:

$$
\beta_{1} \beta_{2} \sim \beta_{1}^{\prime} t_{k, n}^{p_{k}} \sigma_{n}^{ \pm 1} \beta_{2}^{\prime}
$$

for $\beta_{1}, \beta_{2} \in B_{m, n}$, where $\beta_{1}^{\prime}, \beta_{2}^{\prime} \in B_{m, n+1}$ are the words $\beta_{1}, \beta_{2}$ respectively, with the substitutions

$$
\begin{aligned}
& a_{k}^{ \pm 1} \longleftrightarrow\left[\left(\lambda_{n-1}^{-1} \sigma_{n}^{2} \lambda_{n-1}\right) a_{k}\right]^{ \pm 1} \\
& a_{i}^{ \pm 1} \longleftrightarrow\left(\lambda_{n-1}^{-1} \sigma_{n}^{2} \lambda_{n-1}\right) a_{i}^{ \pm 1}\left(\lambda_{n-1}^{-1} \sigma_{n}^{2} \lambda_{n-1}\right)^{-1}, \quad \text { if } i<k \\
& a_{i}^{ \pm 1} \longleftrightarrow a_{i}^{ \pm 1}, \quad \text { if } i>k .
\end{aligned}
$$

Moreover, a combed algebraic band move is a parted band move that is the composition of an algebraic band move with the combing of the parallel strand

$$
\beta_{1} \beta_{2} \sim \beta_{1}^{\prime} t_{k, n}^{p_{k}} \sigma_{n}^{ \pm 1} \beta_{2}^{\prime} r_{k}
$$

where $r_{k}$ is the combing of the parted parallel strand to the $k$ th surgery strand through the surgery braid.

In Figure 20 note that the isotopy of the little band in the dotted box is treated as 'invisible', that is, as identity in the braid group.

Lemma 8. Performing a parted band move on a parted mixed braid and then combing, the result is the same as combing the mixed braid and then performing an algebraic band move.

Proof. The parted band move takes place at the top part of the braid, so it resembles an algebraic band move. Therefore, we just have to consider the behaviour of the parallel strand with respect to combing. On the other hand, the fact that a band move takes place very close to the surgery strand 
Algebraic Markov equivalence for Links in three-manifolds

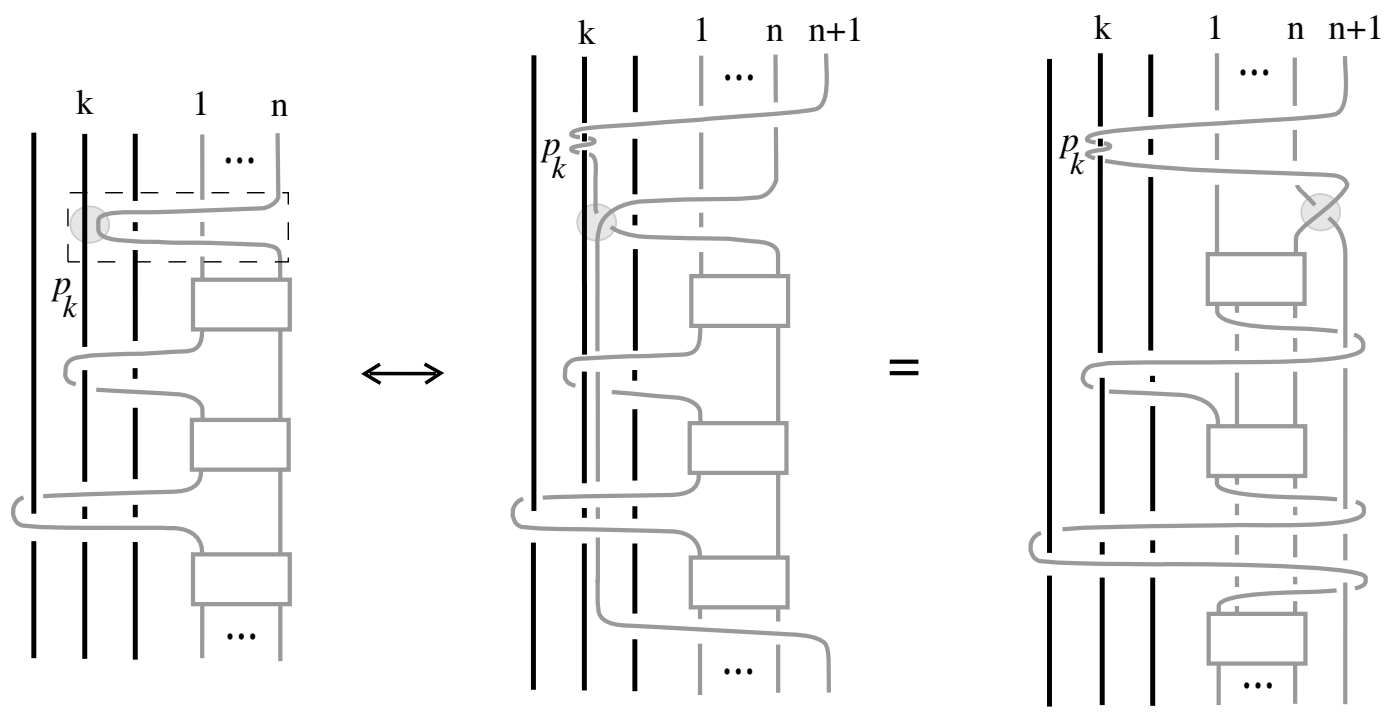

FiguRE 20. An algebraic band move and its algebraic expression.
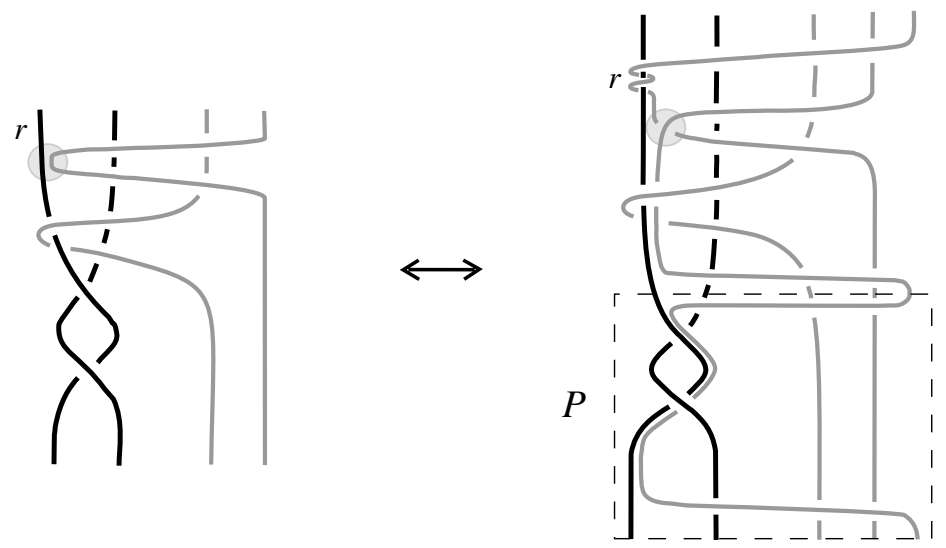

Figure 21. Parted band move $=$ algebraic band move + combing.

ensures that the loops $a_{k}^{ \pm 1}$ around the specific surgery strand get combed in the same way before and after the band move.

So, when we perform a parted band move on a parted mixed braid we comb away all of the loops $a_{k}^{ \pm 1}$ and we leave last the combing of the parallel moving strand. This combing will be the same in either case of the statement of the lemma. In Figure 21 we show that using a small braid isotopy at the bottom of the algebraic part we create an algebraic band move followed by the combing through the dotted box $P$ of the parted parallel strand. Note, finally, that the combing of the parallel strand leaves clear the fixed braid at the bottom. Thus, the proof is concluded.

We are now in the position to state the following result.

Theorem 5 (Algebraic Markov theorem for $\chi\left(S^{3}, \widehat{B}\right)$ ). Two oriented links in $\chi\left(S^{3}, \widehat{B}\right)$ are isotopic if and only if any two corresponding algebraic mixed braid representatives in $B_{m, \infty}$ differ by a finite sequence of the following moves:

(1) algebraic $M$-moves: $\alpha_{1} \alpha_{2} \sim \alpha_{1} \sigma_{n}^{ \pm 1} \alpha_{2}$, for $\alpha_{1}, \alpha_{2} \in B_{m, n}$;

(2) algebraic Markov conjugation: $\alpha \sim \sigma_{j}^{ \pm 1} \alpha \sigma_{j}^{\mp 1}$, for $\alpha, \sigma_{j} \in B_{m, n}$; 


\section{S. Lambropoulou and C. P. Rourke}

(3) twisted loop conjugation: $\beta \sim a_{i}^{\mp 1} \beta \rho_{i}^{ \pm 1}$, for $\beta, a_{i}, \rho_{i} \in B_{m, n}$, where $\rho_{i}$ is the combing of the loop $a_{i}$ through $B$;

(4) combed algebraic band moves: for every $k=1, \ldots, m$ we have

$$
\beta_{1} \beta_{2} \sim \beta_{1}^{\prime} t_{k, n}^{p_{k}} \sigma_{n}^{ \pm 1} \beta_{2}^{\prime} r_{k},
$$

where $\beta_{1}, \beta_{2} \in B_{m, n}$ and $\beta_{1}^{\prime}, \beta_{2}^{\prime} \in B_{m, n+1}$ are as in Definition 7 and where $r_{k}$ is the combing of the parted parallel strand to the $k$ th surgery strand through $B$;

or, equivalently, by a finite sequence of the following moves:

$\left(1^{\prime}\right)$ algebraic $L$-moves (see algebraic expressions in Definition 6);

$\left(2^{\prime}\right)$ twisted loop conjugation;

$\left(3^{\prime}\right)$ Combed algebraic band moves.

Proof. By Theorem 4, we only have to consider the case where a parted band move takes place and, by Theorem 3, we only have to consider the behaviour of a parted band move with respect to combing. This is done in Lemma 8, in the proof of which it is also explained that the combing of the parallel strand gives rise to a combed algebraic band move on the level of $B_{m, \infty}$.

Remark 2. We remark that $t_{k, n}^{p_{k}}$ in Definition 7 of an algebraic band move is just a Markov conjugate of the loop $a_{k}{ }^{p_{k}}$ and that these are the appropriate words for defining inductive Markov traces on quotient algebras of group algebras of $B_{m, n}$. Note also that the words in the parentheses of the substitutions of the loops get significantly simplified if we apply a quadratic relation on the $\sigma_{i}$. Moreover, in Theorem 5 we obtain the best possible control over the band moves of links in closed three-manifolds, and this is very useful for the study of skein modules of closed three-manifolds [Prz06].

\section{Special cases and examples}

In this section we give the braid equivalences described in Theorems 4 and 5 for specific examples of knot complements and closed three-manifolds. We also discuss the adaptation of the band move and braid equivalence for the case where the fixed braid $B$ describing the manifold is not a pure braid, and we study the example where $B$ is the closure of a trefoil.

Example 1. Let $V$ be the solid torus or the lens space $L(p, 1)$, for a framing $p \in \mathbb{Z}$. Then the description of $V$ in $S^{3}$ is the unknot, and so for $t:=a_{1}$ and $t_{n}:=\sigma_{n} \cdots \sigma_{1} t \sigma_{1}^{-1} \cdots \sigma_{n}^{-1}$ we have the following. Two oriented links in a solid torus are isotopic if and only if any two corresponding mixed braids in $B_{1, \infty}$ differ by a finite sequence of the following moves:

(1) algebraic $M$-moves: $\alpha \sim \alpha \sigma_{n}^{ \pm 1}, \alpha \in B_{1, n}$;

(2) algebraic Markov conjugation: $\alpha \sim \sigma_{i}^{\mp 1} \alpha \sigma_{i}^{ \pm 1}, \alpha, \sigma_{i} \in B_{1, n}$;

(3) loop conjugation: $\beta \sim t^{\mp 1} \beta t^{ \pm 1}, \beta \in B_{1, n}$.

Moreover, if the two links lie in $L(p, 1)$ then the corresponding algebraic mixed braids differ by a finite sequence of the above moves together with the following:

(4) algebraic band moves: for $\beta \in B_{1, n}$ we have

$$
\beta \sim t_{n}^{p} \sigma_{n}^{ \pm 1} \beta^{\prime}
$$

where $\beta^{\prime} \in B_{1, n+1}$ is the word $\beta$ with the substitutions

$$
t^{ \pm 1} \longleftrightarrow\left[\left(\lambda_{n-1}^{-1} \sigma_{n}^{2} \lambda_{n-1}\right) t\right]^{ \pm 1} .
$$




\section{Algebraic Markov EQUivalence For Links in THREE-MANifolds}

Remark 3. Constructing all analogues of the two-variable Jones polynomial in the solid torus via braids has been completely studied, see [Lam99] and references therein. These invariants are related to the third skein module of the solid torus [HK90, Tur88]. Moreover, this last move (4) is used by the first author and J. H. Przytycki in order to investigate the third skein module of the lens spaces $L(p, 1)$.

Example 2. Let $V$ be the complement of the $m$-unlink or a connected sum of $m$ lens spaces of type $L(p, 1)$. Then the fixed braid representing $V$ is the identity braid, $I_{m}$, and so we have the following. Two oriented links in the complement of the m-unlink are isotopic if and only if any two corresponding mixed braids in $B_{m, \infty}$ differ by a finite sequence of the following moves:

(1) algebraic $M$-moves: $\alpha \sim \alpha \sigma_{n}^{ \pm 1}, \alpha \in B_{m, n}$;

(2) algebraic Markov conjugation: $\alpha \sim \sigma_{i}^{\mp 1} \alpha \sigma_{i}^{ \pm 1}, \alpha, \sigma_{i} \in B_{m, n}$;

(3) algebraic loop conjugation: $\beta \sim a_{i}^{\mp 1} \beta a_{i}^{ \pm 1}, \beta \in B_{m, n}, i=1, \ldots, m$.

(Compare with [HL02, Theorem 5] about braid equivalence in handlebodies.)

Moreover, if the two links lie in the connected sum $L\left(p_{1}, 1\right) \# \cdots \# L\left(p_{m}, 1\right)$, where $p_{1}, \ldots, p_{m} \in \mathbb{Z}$, then the corresponding algebraic mixed braids differ by a finite sequence of the above moves together with the following:

(4) algebraic band moves: for $\beta \in B_{m, n}$ and for $k=1, \ldots, m$ we have

$$
\beta \sim t_{k, n}^{p_{k}} \sigma_{n}^{ \pm 1} \beta^{\prime}
$$

where $\beta^{\prime} \in B_{m, n+1}$ is the word $\beta$ with the substitutions

$$
\begin{aligned}
& a_{k}^{ \pm 1} \longleftrightarrow\left[\left(\lambda_{n-1}^{-1} \sigma_{n}^{2} \lambda_{n-1}\right) a_{k}\right]^{ \pm 1} \\
& a_{i}^{ \pm 1} \longleftrightarrow\left(\lambda_{n-1}^{-1} \sigma_{n}^{2} \lambda_{n-1}\right) a_{i}^{ \pm 1}\left(\lambda_{n-1}^{-1} \sigma_{n}^{2} \lambda_{n-1}\right)^{-1}, \quad \text { if } i<k \\
& a_{i}^{ \pm 1} \longleftrightarrow a_{i}^{ \pm 1}, \quad \text { if } i>k .
\end{aligned}
$$

Example 3. Let $V$ be the complement of the Hopf link or a lens space $L(p, q)$ obtained by doing surgery along the Hopf link with framings $p_{1}, p_{2} \in \mathbb{Z}$ (obtained from the numerical equation $p / q=$ $\left.p_{1}+1 / p_{2}\right)$. The fixed braid representing $V$ is $\Sigma_{1}^{2}$ and we have the following.

- Relations for the twisted conjugation:

$$
\begin{aligned}
& \Sigma_{1}^{2} \cdot a_{1}^{ \pm 1}=a_{2}^{-1} a_{1}^{ \pm 1} a_{2} \cdot \Sigma_{1}^{2}, \\
& \Sigma_{1}^{2} \cdot a_{2}^{ \pm 1}=a_{2}^{-1} a_{1}^{-1} a_{2}^{ \pm 1} a_{1} a_{2} \cdot \Sigma_{1}^{2} .
\end{aligned}
$$

- Combed algebraic band moves: for $\beta_{1}, \beta_{2} \in B_{2, n}$ and $k=1,2$ we have

$$
\beta_{1} \beta_{2} \sim \beta_{1}^{\prime} t_{k, n}^{p_{k}} \sigma_{n}^{ \pm 1} \beta_{2}^{\prime} r_{k}
$$

where $r_{k}$ is the combing through the fixed braid of the parted moving strand parallel to the $k$ th surgery strand. For $\lambda_{n}:=\sigma_{n} \cdots \sigma_{1}, r_{1}, r_{2}$ are given by the relations:

$$
\begin{aligned}
& r_{1}=\lambda_{n} a_{2} \lambda_{n}^{-1} \\
& r_{2}=\lambda_{n} a_{2}^{-1} a_{1} a_{2} \lambda_{n}^{-1} .
\end{aligned}
$$

The words $\beta_{1}^{\prime}, \beta_{2}^{\prime} \in B_{2, n+1}$ are the words $\beta_{1}, \beta_{2}$ with the following changes, depending on whether the band move is taking place along the first surgery strand or along the second. That is, if $k=1$, then $\beta_{1}^{\prime}, \beta_{2}^{\prime}$ are obtained from $\beta_{1}, \beta_{2}$ by doing the substitutions:

$$
\begin{aligned}
& a_{1}^{ \pm 1} \longleftrightarrow\left[\left(\lambda_{n-1}^{-1} \sigma_{n}^{2} \lambda_{n-1}\right) a_{1}\right]^{ \pm 1} \\
& a_{2}^{ \pm 1} \longleftrightarrow a_{2}^{ \pm 1} .
\end{aligned}
$$




\section{S. Lambropoulou and C. P. Rourke}

If $k=2$, then $\beta_{1}^{\prime}, \beta_{2}^{\prime}$ are obtained from $\beta_{1}, \beta_{2}$ by doing the substitutions:

$$
\begin{aligned}
& a_{1}^{ \pm 1} \longleftrightarrow\left(\lambda_{n-1}^{-1} \sigma_{n}^{2} \lambda_{n-1}\right) a_{1}^{ \pm 1}\left(\lambda_{n-1}^{-1} \sigma_{n}^{2} \lambda_{n-1}\right)^{-1} \\
& a_{2}^{ \pm 1} \longleftrightarrow\left[\left(\lambda_{n-1}^{-1} \sigma_{n}^{2} \lambda_{n-1}\right) a_{2}\right]^{ \pm 1} .
\end{aligned}
$$

Example 4. Now let $V$ be in general the complement of a daisy chain on $m$ rings or a lens space of type $L(p, q)$ obtained by doing surgery along the components, with framings $p_{1}, \ldots, p_{m} \in \mathbb{Z}$ (which are obtained from the continued fraction expansion of the rational number $p / q$ ). The basic manifolds of this series are described in the previous example. A fixed braid representing $V$ is

$$
\Sigma_{1}^{2} \Sigma_{3}^{2} \ldots \Sigma_{2 k-1}^{2} \Sigma_{2}^{2} \Sigma_{4}^{2} \ldots \Sigma_{2 k-2}^{2}:=\mathcal{D} C_{2 k},
$$

if the daisy chain consists of $m=2 k$ rings, and

$$
\Sigma_{1}^{2} \Sigma_{3}^{2} \ldots \Sigma_{2 k-1}^{2} \Sigma_{2}^{2} \Sigma_{4}^{2} \ldots \Sigma_{2 k}^{2}:=\mathcal{D} C_{2 k+1},
$$

if the daisy chain consists of $m=2 k+1$ rings. It is easy to verify the above braid words by closing the odd-numbered strands by simple arcs that run under the braid and the even-numbered strands by simple arcs that run over the braid.

- Relations for the twisted conjugation: we give relations for $2 k$ and $2 k+1$ rings by inductive formulas. For $m=2$ the relations for $a_{1}^{ \pm 1}$ and $a_{2}^{ \pm 1}$ are given in the previous example. For $m=3$ we have the 'twisted' relations:

$$
\begin{aligned}
& {\left[\Sigma_{1}^{2} \Sigma_{2}^{2}\right] \cdot a_{1}^{ \pm 1}=a_{2}^{-1} a_{1}^{ \pm 1} a_{2} \cdot\left[\Sigma_{1}^{2} \Sigma_{2}^{2}\right],} \\
& {\left[\Sigma_{1}^{2} \Sigma_{2}^{2}\right] \cdot a_{2}^{ \pm 1}=\left(a_{1} a_{2} a_{3}\right)^{-1} a_{2}^{ \pm 1}\left(a_{1} a_{2} a_{3}\right) \cdot\left[\Sigma_{1}^{2} \Sigma_{2}^{2}\right],} \\
& {\left[\Sigma_{1}^{2} \Sigma_{2}^{2}\right] \cdot a_{3}^{ \pm 1}=\left(a_{2}^{-1} a_{1}^{-1} a_{2} a_{1} a_{2} a_{3}\right)^{-1} a_{3}^{ \pm 1}\left(a_{2}^{-1} a_{1}^{-1} a_{2} a_{1} a_{2} a_{3}\right) \cdot\left[\Sigma_{1}^{2} \Sigma_{2}^{2}\right] .}
\end{aligned}
$$

Note that the relation for $a_{1}^{ \pm 1}$ is the same as for two rings.

Suppose now that the twisted conjugation relations are known for $2 k-2$ and $2 k-1$ rings and consider $2 k$ rings. For $1 \leqslant i \leqslant 2 k-3$ the twisted relations for $\mathcal{D} C_{2 k} \cdot a_{i}^{ \pm 1}$ are the same as those for $\mathcal{D} C_{2 k-2} \cdot a_{i}^{ \pm 1}$. For $i=2 k-2,2 k-1,2 k$ we have the following relations, that are easy consequences of Lemma 6 :

$$
\begin{aligned}
\mathcal{D} C_{2 k} \cdot a_{2 k-2}^{ \pm 1}= & \left(a_{2 k-3} a_{2 k-2} a_{2 k}^{-1} a_{2 k-1} a_{2 k}\right)^{-1} a_{2 k-2}^{ \pm 1}\left(a_{2 k-3} a_{2 k-2} a_{2 k}^{-1} a_{2 k-1} a_{2 k}\right) \cdot \mathcal{D} C_{2 k}, \\
\mathcal{D} C_{2 k} \cdot a_{2 k-1}^{ \pm 1}= & \left(a_{2 k} a_{2 k-2}^{-1} a_{2 k-3}^{-1} a_{2 k-2} a_{2 k-3} a_{2 k-2} a_{2 k}^{-1} a_{2 k-1} a_{2 k}\right)^{-1} \\
& \cdot\left(a_{2 k} a_{2 k-2}^{-1} a_{2 k-3}^{-1} a_{2 k-2} a_{2 k-3} a_{2 k-2} a_{2 k}^{-1} a_{2 k-1} a_{2 k}\right) \cdot \mathcal{D} C_{2 k}, \\
\mathcal{D} C_{2 k} \cdot a_{2 k}^{ \pm 1}= & \left(a_{2 k-1} a_{2 k}\right)^{-1} a_{2 k}^{ \pm 1}\left(a_{2 k-1} a_{2 k}\right) \cdot \mathcal{D} C_{2 k} .
\end{aligned}
$$

Finally, if we have $2 k+1$ rings, then for $1 \leqslant i \leqslant 2 k-1$ the twisted relations for $\mathcal{D} C_{2 k+1} \cdot a_{i}^{ \pm 1}$ are the same as those for $\mathcal{D} C_{2 k} \cdot a_{i}^{ \pm 1}$. For $i=2 k, 2 k+1$ we have the following relations, that are also easy consequences of Lemma 6 :

$$
\begin{aligned}
\mathcal{D} C_{2 k+1} \cdot a_{2 k}^{ \pm 1}= & \left(a_{2 k-1} a_{2 k} a_{2 k+1}\right)^{-1} a_{2 k}^{ \pm 1}\left(a_{2 k-1} a_{2 k} a_{2 k+1}\right) \cdot \mathcal{D} C_{2 k+1}, \\
\mathcal{D} C_{2 k+1} \cdot a_{2 k+1}^{ \pm 1}= & \left(a_{2 k}^{-1} a_{2 k-1}^{-1} a_{2 k} a_{2 k-1} a_{2 k} a_{2 k+1}\right)^{-1} a_{2 k+1}^{ \pm 1} \\
& \cdot\left(a_{2 k}^{-1} a_{2 k-1}^{-1} a_{2 k} a_{2 k-1} a_{2 k} a_{2 k+1}\right) \cdot \mathcal{D} C_{2 k+1} .
\end{aligned}
$$

- Combed algebraic band moves: for $\beta_{1}, \beta_{2} \in B_{m, n}$ and for $s=1, \ldots, m$ we have

$$
\beta_{1} \beta_{2} \sim \beta_{1}^{\prime} t_{s, n}^{p_{s}} \sigma_{n}^{ \pm 1} \beta_{2}^{\prime} r_{s}
$$




\section{Algebraic Markov equivalence For Links in three-Manifolds}

where $\beta_{1}^{\prime}, \beta_{2}^{\prime} \in B_{m, n+1}$ are the words $\beta_{1}, \beta_{2}$ with the substitutions

$$
\begin{aligned}
& a_{s}^{ \pm 1} \longleftrightarrow\left[\left(\lambda_{n-1}^{-1} \sigma_{n}^{2} \lambda_{n-1}\right) a_{s}\right]^{ \pm 1} \\
& a_{i}^{ \pm 1} \longleftrightarrow\left(\lambda_{n-1}^{-1} \sigma_{n}^{2} \lambda_{n-1}\right) a_{i}^{ \pm 1}\left(\lambda_{n-1}^{-1} \sigma_{n}^{2} \lambda_{n-1}\right)^{-1}, \quad \text { if } i<s \\
& a_{i}^{ \pm 1} \longleftrightarrow a_{i}^{ \pm 1}, \quad \text { if } i>s
\end{aligned}
$$

and where $r_{s} \in B_{m, n+1}$ is the combing through $\mathcal{D} C_{m}$ of the parted moving strand parallel to the $s$ th surgery strand. For any index $m$ of $\mathcal{D} C_{m}$ the combings $r_{1}, \ldots, r_{m}$ are given by the following relations:

$$
r_{1}=\lambda_{n} a_{2} \lambda_{n}^{-1} .
$$

The combings $r_{2}, \ldots, r_{m-2}$ are given by the following paired formulas:

$$
\begin{aligned}
r_{2 k} & =\lambda_{n}\left(a_{2 k}^{-1} a_{2 k-1} a_{2 k} a_{2 k+2}^{-1} a_{2 k+1} a_{2 k+2}\right) \lambda_{n}^{-1} \\
r_{2 k+1} & =\lambda_{n}\left(a_{2 k+1}^{-1} a_{2 k+2} a_{2 k}^{-1} a_{2 k-1}^{-1} a_{2 k} a_{2 k-1} a_{2 k} a_{2 k+2}^{-1} a_{2 k+1} a_{2 k+2}\right) \lambda_{n}^{-1} .
\end{aligned}
$$

The final combings $r_{m-1}, r_{m}$ depend on whether $m$ is even or odd. For $m$ even we have

$$
\begin{aligned}
r_{m-1} & =\text { as above for odd index } \\
r_{m} & =\lambda_{n}\left(a_{m}^{-1} a_{m-1} a_{m}\right) \lambda_{n}^{-1} .
\end{aligned}
$$

For $m$ odd we have

$$
\begin{aligned}
r_{m-1} & =\lambda_{n}\left(a_{m-1}^{-1} a_{m-2} a_{m-1} a_{m}\right) \lambda_{n}^{-1} \\
r_{m} & =\lambda_{n}\left(a_{m}^{-1} a_{m-1}^{-1} a_{m-2}^{-1} a_{m-1} a_{m-2} a_{m-1} a_{m}\right) \lambda_{n}^{-1} .
\end{aligned}
$$

Example 5. Let $V$ be the complement of the Borromean rings or a closed manifold obtained by doing surgery along them, with framings $p_{1}, p_{2}, p_{3} \in \mathbb{Z}$. In particular, with framings +1 we obtain dodecahedral space. The fixed braid representing $V$ is

$$
\Sigma_{1}^{-1} \Sigma_{2} \Sigma_{1}^{-1} \Sigma_{2} \Sigma_{1}^{-1} \Sigma_{2}:=\mathcal{B} R \text {. }
$$

- Relations for the twisted conjugation:

$$
\begin{aligned}
\mathcal{B} R \cdot a_{1}^{ \pm 1}= & \left(a_{3} a_{1} a_{2} a_{1}^{-1} a_{3}^{-1} a_{1} a_{2}^{-1} a_{1}^{-1}\right)^{-1} a_{1}^{ \pm 1}\left(a_{3} a_{1} a_{2} a_{1}^{-1} a_{3}^{-1} a_{1} a_{2}^{-1} a_{1}^{-1}\right) \cdot \mathcal{B} R \\
\mathcal{B} R \cdot a_{2}^{ \pm 1}= & \left(a_{1}^{-1} a_{3}^{-1} a_{1} a_{3}\right)^{-1} a_{2}^{ \pm 1}\left(a_{1}^{-1} a_{3}^{-1} a_{1} a_{3}\right) \cdot \mathcal{B} R \\
\mathcal{B} R \cdot a_{3}^{ \pm 1}= & \left(a_{1} a_{2}^{-1} a_{1}^{-1} a_{3}^{-1} a_{1}^{-1} a_{3} a_{1} a_{2} a_{1}^{-1} a_{3}^{-1} a_{1} a_{3}\right)^{-1} a_{3}^{ \pm 1} \\
& \cdot\left(a_{1} a_{2}^{-1} a_{1}^{-1} a_{3}^{-1} a_{1}^{-1} a_{3} a_{1} a_{2} a_{1}^{-1} a_{3}^{-1} a_{1} a_{3}\right) \cdot \mathcal{B} R .
\end{aligned}
$$

- Combed algebraic band moves: for $\beta_{1}, \beta_{2} \in B_{3, n}$ and for $k=1,2,3$ we have

$$
\beta_{1} \beta_{2} \sim \beta_{1}^{\prime} t_{k, n}^{p_{k}} \sigma_{n}^{ \pm 1} \beta_{2}^{\prime} r_{k}
$$

where $\beta_{1}^{\prime}, \beta_{2}^{\prime} \in B_{3, n+1}$ are the words $\beta_{1}, \beta_{2}$ with the substitutions

$$
\begin{aligned}
& a_{k}^{ \pm 1} \longleftrightarrow\left[\left(\lambda_{n-1}^{-1} \sigma_{n}^{2} \lambda_{n-1}\right) a_{k}\right]^{ \pm 1} \\
& a_{i}^{ \pm 1} \longleftrightarrow\left(\lambda_{n-1}^{-1} \sigma_{n}^{2} \lambda_{n-1}\right) a_{i}^{ \pm 1}\left(\lambda_{n-1}^{-1} \sigma_{n}^{2} \lambda_{n-1}\right)^{-1}, \quad \text { if } i<k \\
& a_{i}^{ \pm 1} \longleftrightarrow a_{i}^{ \pm 1}, \quad \text { if } i>k
\end{aligned}
$$

and where $r_{k} \in B_{3, n+1}$ is the combing through $\mathcal{B} R$ of the parted moving strand parallel to the $k$ th surgery strand. The combings $r_{1}, r_{2}, r_{3}$ are given by the following relations, which are easy 


\section{S. Lambropoulou and C. P. Rourke}
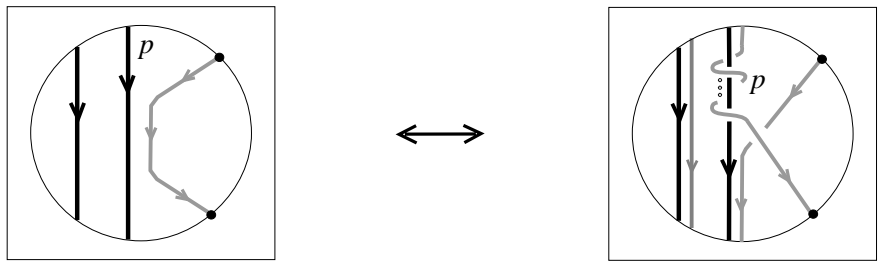

FiguRE 22. The band move for a non-pure surgery braid.
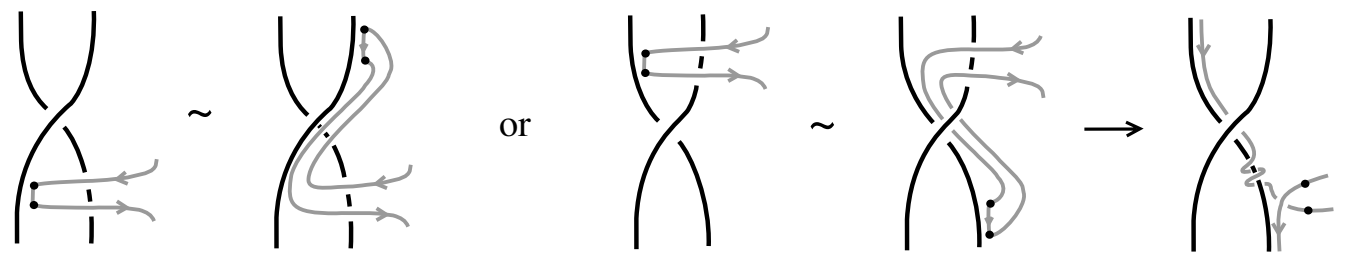

Figure 23. Passing the little band to the right.

consequences of Lemma 6.

$$
\begin{aligned}
& r_{1}=\lambda_{n}\left(a_{3} a_{1} a_{2} a_{1}^{-1} a_{3}^{-1} a_{1} a_{2}^{-1} a_{1}^{-1}\right) \lambda_{n}^{-1}, \\
& r_{2}=\lambda_{n}\left(a_{1}^{-1} a_{3}^{-1} a_{1} a_{3}\right) \lambda_{n}^{-1}, \\
& r_{1}=\lambda_{n}\left(a_{1} a_{2}^{-1} a_{1}^{-1} a_{3}^{-1} a_{1}^{-1} a_{3} a_{1} a_{2} a_{1}^{-1} a_{3}^{-1} a_{1} a_{3}\right) \lambda_{n}^{-1} .
\end{aligned}
$$

\section{The case of non-pure surgery braids}

A closed three-manifold may be easier described by a non-pure surgery braid. As noted in [LR97, Remark 5.11], in this case Theorem 1 and consequently Theorems 3 and 5 still apply, but now the band moves are more complicated to express: in this case a band move is modified so that the replacement of the little band only twists around one of the strands of the same surgery component and it runs in parallel to all other strands of that surgery component. See Figure 22. In Lemma 9 below we show that such a band move may be always assumed to have a specific form. We part such a band move at the top by pulling all parallel strands to the last positions of the moving part, over all strands in between and respecting their order.

Lemma 9. If the surgery braid $B$ is not a pure braid, then, up to $L$-equivalence, a band move may always be assumed to take place at the top part of the braid and on the right of the rightmost strand of the specific surgery component.

Moreover, performing a band move at the top part of a mixed braid and then parting, yields the same, up to L-equivalence and loop conjugation, as performing a parted band move on a parted mixed braid.

Proof. Assume that the little band does not attach to the rightmost strand of the specific surgery component. Then, as in the proof of Lemma 5, stretch the little band and its replacement in parallel to the surgery strand and to the top or to the bottom of the mixed braid, depending on which direction brings it to the right. See Figure 23.

If we have landed at the bottom we do appropriate $L$-moves to transfer the crossing of the band move to the top. Recall Figure 14. If we have reached the rightmost strand we stop. If not, we continue sliding the little band and its replacement until the rightmost strand is reached. Similarly, by loop conjugations we bring the framing twists to the top of the rightmost strand of the specific component. 


\section{Algebraic Markov EQUivalence For Links in THREE-MANifolds}

If, moreover, the two mixed braids that differ by the band move are parted we apply the same strategy as in the proof of Lemma 5 but adapted here.

Example 6. As an application of the above lemma consider $V$ to be the complement of the righthanded trefoil or a closed manifold obtained by doing surgery along it with framing $k \in \mathbb{Z}$. In particular, with framing -1 we obtain the dodecahedral space. The fixed braid representing $V$ is $\Sigma_{1}^{3}$ and we have the following.

- Relations for the twisted conjugation:

$$
\begin{aligned}
& \Sigma_{1}^{3} \cdot a_{1}^{ \pm 1}=\left(a_{1} a_{2}\right)^{-1} a_{2}^{ \pm 1}\left(a_{1} a_{2}\right) \cdot \Sigma_{1}^{3} \\
& \Sigma_{1}^{3} \cdot a_{2}^{ \pm 1}=\left(a_{2} a_{1} a_{2}\right)^{-1} a_{1}^{ \pm 1}\left(a_{2} a_{1} a_{2}\right) \cdot \Sigma_{1}^{3}
\end{aligned}
$$

- Combed algebraic band moves: for $\beta_{1}, \beta_{2} \in B_{2, n}$ we have

$$
\beta_{1} \beta_{2} \sim \beta_{1}^{\prime} \sigma_{n+1}^{-1} t_{2, n}^{k} \sigma_{n}^{ \pm 1} \sigma_{n+1} \beta_{2}^{\prime} r
$$

where $r=\lambda_{n}\left(a_{2}\right) \sigma_{n+1} \lambda_{n}\left(a_{2}^{-1} a_{1} a_{2}\right) \sigma_{1}^{2} \lambda_{n}^{-1} \sigma_{n+1}^{-1}\left(a_{2}^{-1} a_{1}^{-1} a_{2} a_{1} a_{2}\right) \lambda_{n}^{-1} \sigma_{n+1} \quad$ is the combing through the fixed braid of the parted moving strands parallel to the two surgery strands, $t_{2, n}=\lambda_{n} a_{k} \lambda_{n}^{-1}$, and where $\beta_{1}^{\prime}, \beta_{2}^{\prime} \in B_{2, n+2}$ are the words $\beta_{1}, \beta_{2}$ with the substitutions

$$
\begin{aligned}
& a_{1}^{ \pm 1} \longleftrightarrow\left(\left[\lambda_{n-1}^{-1}\left(\sigma_{n} \sigma_{n+1}^{2} \sigma_{n}\right) \lambda_{n-1}\right] a_{1}\left[\lambda_{n-1}^{-1}\left(\sigma_{n} \sigma_{n+1}^{2} \sigma_{n}^{-1}\right)^{-1} \lambda_{n-1}\right]\right)^{ \pm 1}, \\
& a_{2}^{ \pm 1} \longleftrightarrow\left(\left[\lambda_{n-1}^{-1}\left(\sigma_{n} \sigma_{n+1}^{2} \sigma_{n}^{-1}\right) \lambda_{n-1}\right] a_{2}\right)^{ \pm 1} .
\end{aligned}
$$

\section{REFERENCES}

Ale23 J. W. Alexander, A lemma on systems of knotted curves, Proc. Natl. Acad. Sci. USA 9 (1923), 93-95.

Bir74 J. S. Birman, Braids, links and mapping class groups, Annals of Mathematics Studies, vol. 82 (Princeton University Press, Princeton, NJ, 1974).

Bru97 H. Brunn, Über verknotete Curven, Verh. Intern. Math. Congr. 1 (1897), 256-259.

HK90 J. Hoste and M. Kidwell, Dichromatic link invariants, Trans. Amer. Math. Soc. 321 (1990), 197-229. (See also the preliminary version of this paper Invariants of colored links, Preprint (1987).)

HL02 R. Häring-Oldenburg and S. Lambropoulou, Knot theory in handlebodies, J. Knot Theory Ramifications 11 (2002), 921-943.

Jon87 V. F. R. Jones, Hecke algebra representations of braid groups and link polynomials, Ann. of Math. (2) 126 (1987), 335-388.

Kir78 R. Kirby, A Calculus for Framed Links in $S^{3}$, Invent. Math. 45 (1978), 35-56.

KS92 K. H. Ko and L. Smolinsky, The framed braid group and 3-manifolds, Proc. Amer. Math. Soc. 115 (1992), 541-551.

Lam99 S. Lambropoulou, Knot theory related to generalized and cyclotomic Hecke algebras of type B, J. Knot Theory Ramifications 8 (1999), 621-658.

Lam00 S. Lambropoulou, Braid structures in handlebodies, knot complements and 3-manifolds, in Proceedings of Knots in Hellas '98, Series of Knots and Everything, vol. 24 (World Scientific Press, Singapore, 2000), 274-289.

LR97 S. Lambropoulou and C. P. Rourke, Markov's theorem in 3-manifolds, Topology Appl. 78 (1997), $95-122$.

Lic62 W. B. R. Lickorish, A representation of orientable combinatorial 3-manifolds, Ann. of Math. (2) 76 (1962), 531-538.

Mar35 A. A. Markov, Über die freie Äquivalenz geschlossener Zöpfe, Recusil Math. Moscou 1 (1935).

Prz06 J. H. Przytycki, Skein modules, Preprint (2006), math.GT/0602264. 
RS72 C. P. Rourke and B. J. Sanderson, Introduction to piecewise linear topology (Springer, Berlin, 1972).

Tur88 V. G. Turaev, The Conway and Kauffman modules of the solid torus, Zap. Nauchn. Sem. LOMI 167 (1988), 79-89. (English transl. J. Soviet Math. 52 (1990), no. 1, 2799-2805.)

Wal60 A. D. Wallace, Modifications and cobounding manifolds, Canad. J. Math. 12 (1960), 503-528.

Wei39 N. Weinberg, Sur l'équivalence libre des tresses fermées, C. R. (Dokl.) Acad. Sci. URSS 23 (1939), 215-216.

S. Lambropoulou sofia@math.ntua.gr, http://www.math.ntua.gr/ sofia

Department of Mathematics, National Technical University of Athens, Zografou Campus, GR-157 80 Athens, Greece

C. P. Rourke cpr@maths.warwick.ac.uk, http://www. maths.warwick.ac.uk/ cpr

Department of Mathematics, University of Warwick, Coventry CV4 7AL, UK 